\title{
The Subjective Value of Cognitive Effort is Encoded by a Domain-General Valuation Network
}

\author{
(D)Andrew Westbrook, ${ }^{1,2,3}$ Bidhan Lamichhane, ${ }^{4}$ and Todd Braver ${ }^{4,5,6}$ \\ ${ }^{1}$ Donders Institute for Brain, Cognition and Behaviour, Radboud University, 6525 EN Nijmegen, The Netherlands, ${ }^{2}$ Department of Psychiatry, Radboud \\ University Medical Centre, Nijmegen, The Netherlands, ${ }^{3}$ Department of Cognitive, Linguistics, and Psychological Sciences, Brown University, Providence, \\ Rhode Island 02912, ${ }^{4}$ Department of Psychological and Brain Sciences, Washington University in St. Louis, St. Louis, Missouri 63130, Departments of \\ ${ }^{5}$ Radiology, and ${ }^{6}$ Neuroscience, Washington University in St. Louis, School of Medicine, St. Louis, Missouri 63110
}

Cognitive control is necessary for goal-directed behavior, yet people treat cognitive control demand as a cost, which discounts the value of rewards in a similar manner as other costs, such as delay or risk. It is unclear, however, whether the subjective value (SV) of cognitive effort is encoded in the same putatively domain-general brain valuation network implicated in other cost domains, or instead engages a distinct frontoparietal network, as implied by recent studies. Here, we provide rigorous evidence that the valuation network, with core foci in the ventromedial prefrontal cortex and ventral striatum, also encodes SV during cognitive effort-based decision-making in healthy, male and female adult humans. We doubly dissociate this network from frontoparietal regions that are instead recruited as a function of decision difficulty. We show that the domain-general valuation network jointly and independently encodes both reward benefits and cognitive effort costs. We also demonstrate that cognitive effort SV signals predict choice and are influenced by state and trait motivation, including sensitivity to reward and anticipated task performance. These findings unify cognitive effort with other cost domains, and suggest candidate neural mechanisms underlying state and trait variation in willingness to expend cognitive effort.

Key words: cognitive control; cognitive effort; decision-making; motivation; subjective value; vmPFC

\section{Significance Statement}

Subjective effort costs are increasingly understood to diminish cognitive control over task performance and can thus undermine functioning across health and disease. Yet, we are only beginning to understand how decisions about cognitive effort are made. A key question is how subjective values are computed. Recent work suggests that the value of cognitive effort might be computed by networks that are distinct from those involved in other domains like intertemporal and risky decision-making, implying distinct mechanisms. Here we demonstrate that the domain-general network also encodes effort-discounted value, linking cognitive effort closely with other domains. Our results thus elucidate key mechanisms supporting decisions about cognitive effort, and point to candidate neural targets for intervention in disorders involving impaired cognitive motivation.

\section{Introduction}

Cognitive control is necessary for goal-directed behavior (Miller, 2000; Botvinick et al., 2001). Yet, intriguingly, control demands are treated as if they are subjectively costly: people even forgo rewards to avoid them (Kool et al., 2010; Dixon and Christoff, 2012; Westbrook et al., 2013). Subjectively high effort costs have

\footnotetext{
Received Dec. 6, 2018; revised Feb. 25, 2019; accepted March 3, 2019.

Author contributions: A.W. and T.B. designed research; A.W. performed research; A.W. contributed unpublished reagents/analytic tools; A.W., B.L., and T.B. analyzed data;A.W. wrote the first draft of the paper; A.W., B.L., and T.B. edited the paper; A.W. wrote the paper.

This work was supported by Grants R01 AG043461 and R21 AG058206 to T.B., and NIMH Grant 1F31 MH100855-01 to A.W. We thank Sarah Adams in the Cognitive Control and Psychopathology Laboratory at Washington University in St. Louis for assistance with data collection.

The authors declare no competing financial interests.

Correspondence should be addressed to Andrew Westbrook at andrew.westbrook@brown.edu.

https://doi.org/10.1523/JNEUROSCI.3071-18.2019

Copyright $\odot 2019$ the authors
}

clinical implications, undermining functioning in schizophrenia (Gold et al., 2015; Culbreth et al., 2016), ADHD (Volkow et al., 2011), depression (Cohen et al., 2001), and Parkinson's disease (Sinha et al., 2013; Manohar et al., 2015). It is thus critical to identify mechanisms of cognitive effort valuation, as a first step toward targeted clinical interventions that address impaired cognitive motivation.

Decision-making may involve "common currency" subjective value (SV) representations that integrate costs and benefits (Padoa-Schioppa, 2011) and facilitate exchange across choice dimensions (Rangel et al., 2008). Numerous studies have implicated a core valuation network that encodes SV across multiple domains (e.g., risk and delay), with primary foci in the ventromedial prefrontal cortex (vmPFC) and ventral striatum (VS; Levy and Glimcher, 2012; Bartra et al., 2013). Although this network was recently implicated in physical effort-based SV encoding 
(Hogan et al., 2018; Aridan et al., 2019), it has not been demonstrated to encode SV for choices about cognitive effort.

Surprisingly, the only two prior studies investigating SV encoding during cognitive effort-based decision-making (Massar et al., 2015; Chong et al., 2017) did not implicate the core valuation network. Instead, they identified frontoparietal regions that are components of brain networks more typically associated with cognitive control itself: the dorsal anterior cingulate cortex (dACC), dorsolateral prefrontal cortex (dlPFC), and intraparietal sulcus (IPS). Thus, a plausible interpretation is that these regions may be recruited to support the control demands associated with difficult decision-making, such as when options are close in value (Pochon et al., 2008; Shenhav et al., 2014). Their involvement is also consistent with a comparator hypothesis by which regions like the ACC track the differences in chosen versus unchosen offer SV (Hunt et al., 2012; Klein-Flügge et al., 2016).

The fact that these prior studies found no evidence of SV encoding in the core valuation network, however, also raises the possibility that cognitive effort-based decision-making involves different mechanisms than other domains. For example, decisions about cognitive effort might not involve SV computation per se, but rather prospective simulation of task demands to estimate controllability, and opportunity costs with respect to an average reward-rate baseline (cf. Kurzban et al., 2013; Boureau et al., 2015; Shenhav et al., 2017; Musslick et al., 2018).

Alternatively, the prior cognitive effort studies may have simply used methods which detected regions as a function of choice difficulty rather than single offer SV. In particular, both studies tested for SV encoding during simultaneous presentation of competing offers. Thus, any subtle trial features prompting more intensive deliberation as a function of SV might evoke frontoparietal activity (Jimura et al., 2018) that is correlated with SV regressors. Hence, it is critical to complement these approaches with methods that dissociate SV representations from decision difficulty.

In the present study, we used a cognitive effort discounting task (COGED; Westbrook et al., 2013), in conjunction with fMRI, to test whether a domain-general valuation network encodes the SV of cognitive effort. Specifically, we test whether a priori regions-of-interest (ROIs) encode SV during evaluation of single offers while explicitly controlling for decision difficulty. Additionally, to provide more rigorous evidence for SV encoding, we identify valuation network regions that track both the costs (cognitive load) and benefits (reward magnitude) of outcomes, along with subjective measures that may reflect stable, trait differences as well as context-specific (i.e., trial-by-trial) state differences.

\section{Materials and Methods}

Participants. Twenty-one healthy, right-handed, volunteer participants (11 females, mean age $=21$ years) recruited from the local St. Louis community, gave informed consent as prescribed by the Institutional Review Board at Washington University. Before the current imaging study, all participants previously performed a separate behavioral and imaging study focused on $\mathrm{N}$-back task performance. Participant selection for the current study was based on ensuring both uniformly high $\mathrm{N}$-back performance and sufficient individual variability in cognitive effort discounting in the included sample. Compensation for participation was provided at the rate of $\$ 25 / \mathrm{h}$ (plus additional bonus for repeating N-back tasks, based on offers they accept as described in the Experimental Design section).

Apparatus and stimuli. Stimuli were presented using the MATLAB (MathWorks) Psychophysics toolbox v3.0.11 (RRID:SCR_002881; http://www.psychtoolbox.com; Brainard, 1997). For each N-back load, 2 runs of 64 lower-case consonants were presented in 32 point Arial font, in one of six colors corresponding to the $N$-back load level: black [rgb code: $(0,0,0)]$, red $(240,0,0)$, blue $(0,0,255)$, purple $(95,0,115)$, green $(0,110,0)$, and brown $(102,51,0)$ for the 1-6-back, respectively. Load levels were labeled by different colors, rather than by numeric load (" $N$ "), to avoid anchoring confounds during decision-making (Chapman and Johnson, 1999). MR images were collected using a 12-channel 3-T Siemens Trio scanner (Siemens Medical Solutions).

Imaging parameters and acquisition. High-resolution anatomical images were acquired for anatomical references using an MPRAGE sequence $[$ repetition time $(\mathrm{TR})=2400 \mathrm{~ms}$, echo time $(\mathrm{TE})=3.08 \mathrm{~ms}$, flip angle $=8^{\circ}$, inversion time $=1000 \mathrm{~ms}$, voxel size $=1 \times 1 \times 1 \mathrm{~mm}^{3}$ ]. The functional scans were acquired with $\mathrm{T} 2{ }^{\star}$-weighted gradient echoplanar imaging sequence, TR: $2000 \mathrm{~ms}$; TE: $27 \mathrm{~ms}$; flip angle, $90^{\circ}$; field-of-view, $256 \times 256 \mathrm{~mm}^{2}$; dimensions, $64 \times 64$ and 32 axial slices each $4 \mathrm{~mm}$ thick acquired (voxel dimensions: $4 \times 4 \times 4 \mathrm{~mm}^{3}$ ).

\section{Experimental design and statistical analysis}

Procedure and task design. The study was implemented in three phases. In the first phase, following consent and MR compatibility screening, participants performed the $\mathrm{N}$-back task, completing two runs of each load level, in order of increasing load, to familiarize participants with the subjective demands of each level before decision-making. In the second phase, indifference points were estimated, according to a discounting procedure detailed previously (Westbrook et al., 2013). Specifically, participants made a series of choices between repeating a block of one of the higher $N$-back load levels $(N=2-6)$ for a larger payment amount $(\$ 2$, $\$ 3$, or $\$ 4$ ) or instead performing a 1-back block for a lower amount, with the amount offered for the 1-back varied in a stepwise titration procedure (over 5 choices per amount-load pair) until participants were indifferent between the two offers. The indifference point is critical because it quantifies the SV of an offer, discounted by the cognitive effort cost of the higher $N$-back levels relative to the 1-back. For instance, if a participant was indifferent between $\$ 3$ for the 4-back, and $\$ 1.70$ for the 1-back, this indicates that the participant found the additional subjective effort of the 4-back versus the 1-back to cost $\$ 1.30$. In this example, therefore, we consider the SV of both $\$ 1.70$ for the 1-back, and also $\$ 3$ for the 4 -back to be $\$ 1.70$.

In the third phase, which is the focus of this report, participants were scanned while performing a series of decision trials that systematically and orthogonally varied by both decision difficulty and SV of the highload offer. Over the course of 150 trials ( 3 amounts $\times 5$ loads $\times 10$ repetitions), we manipulated the SV of the high-load offer by giving participants choices to either perform one of five, high-load $N$-back levels $(N=2-6)$ for one of three higher reward amounts $(\$ 2, \$ 3$, or $\$ 4)$, and the 1-back at a lower, variable amount. To manipulate decision difficulty, we varied the amount offered for the 1-back according to a proximity parameter, $\gamma$. This parameter specified the difference in estimated SV of the two options, with small absolute values indicating that alternatives were close in SV, and positive values indicating higher SV for the 1-back option. Specifically, $\gamma$ gives the proportion difference between indifference $(\gamma=0)$ and the bounds: e.g., $\gamma=-0.4$ indicates that the offer for the easy task is $40 \%$ below the indifference point, whereas $\gamma=0.2$ indicates that the offer for the easy task is above the indifference point by $20 \%$ of the difference between the indifference point and the amount offered for the hard task. The proximity parameter $\gamma$ was repeated twice for each value from the set $\{-0.4,-0.1,0.2,0.6\}$ yielding 120 regular trials, and once each from the set $\{-1.0,1.0\}$ yielding 30 catch trials. The "catch" trials $(\gamma=1.0$ or -1.0$)$ were referred to this way because they involve obvious choices: either the same amount is offered for the easy and the hard task $(\gamma=1.0)$, which should strongly bias selection of the easier task, or the easy task is associated with $\$ 0$ payment $(\gamma=-1.0)$, which should strongly bias the harder task.

The proximity parameter thus accomplishes three goals. First, it permits testing for decision difficulty encoding by, e.g., contrasting difficult trials in which the proximity parameter is small $(|\gamma| \leq 0.6)$ with easy trials when the parameter is large $(|\gamma|=1.0)$. Second, the specific values of the proximity parameters were designed (based on prior behavioral piloting results) to ensure that participants are likely to select the harder and 
easier tasks in equal proportion. As a consequence, it protects against decision biases, and decisions being anticipated in advance of both options being presented while also ensuring that decision difficulty is balanced across trials and participants. Third, BOLD signal may be contrasted according to whether participants make decisions that are in line with their own indifference points from the second phase to see how trial-level choice patterns are reflected in BOLD signal variation. For example, we might infer that participants had higher momentary motivation on a trial in which they choose the harder alternative, even though we offered more for the easy task than the SV of the hard task. This hypothesis would be supported if we found that BOLD signals encoding SV happened to be augmented on those trials, indicating that trialwise motivation is reflected in SV representations.

Each trial lasted $13 \mathrm{~s}$ (see Fig. 3). All decision trials began with a single high-load offer presented in isolation for $6 \mathrm{~s}$, followed by presentation of both offers (high- and low-load) presented for up to $5.25 \mathrm{~s}$, or until a decision was made. Presenting the high-load option alone enabled us to isolate SV encoding for that offer and avoid any ambiguities about which offer, or combination of offers was encoded from moment-to-moment. Our key test was whether the SV of the high-load option was encoded at the end of this first-offer valuation window. Specifically, we focused on the brain response 6-8 s after first offer onset to isolate encoding of first offer SV, accounting for hemodynamic lag (Miezin et al., 2000). BOLD dynamics pursuant to second offer onset were used only to investigate decision-difficulty effects. Trials were concluded with either a fixationcross, presented after the response was made, or instead, feedback that the response deadline was missed, followed by a fixation cross until the next trial. Trials in which participants did not respond in time $(1.15 \%$ of trials overall) were omitted from further analyses.

Data were collected in two cohorts. In an early cohort (7 participants), $\mathrm{T} 1$ and $\mathrm{T} 2$ anatomical images were collected first, after which all decision trials were completed in a single run while 1019 functional volumes were collected. In the later cohort, to reduce discomfort, trials were broken down into three runs ( 345 volumes each); in between task runs, participants were invited to relax, motionless while $\mathrm{T} 1$ and $\mathrm{T} 2$ anatomical scans were acquired. After all images were collected, one decision trial was chosen at random, and participants were required to repeat one run of the chosen $N$-back level, after which they received the associated reward amount, as a monetary bonus added to their base compensation.

Imaging analyses. All DICOM images were converted to NIFTI format using the FreeSurfer function mri_convert (RRID:SCR_001847; http:// surfer.nmr.mgh.harvard.edu). Subsequent steps of the analysis were implemented with AFNI software functions (RRID:SCR_005927; http:// afni.nimh.nih.gov/afni; Cox, 1996). Brain tissue was masked using the 3dSkullstrip function, images were concatenated using $3 \mathrm{dTcat}$, aligned from oblique to cardinal orientation using the $3 \mathrm{dWarp}$ function, and then up-sampled to $3 \times 3 \times 3 \mathrm{~mm}^{3}$ voxels and aligned across all functional runs to the first run. Parameters for registration of functional volumes with anatomical $\mathrm{T} 1$ images were computed for each participant separately. Precise registration was verified visually for every participant and cost functions were tailored to optimize registration for each participant. Then, parameters for warping participant-specific anatomical images to a standard MNI space (MNI152_T1_2009c+tlrc; Fonov et al., 2011) were computed. All registration and warping parameters were concatenated using the cat_matvec function, and applied as a single transformation to aligned functional image volumes using the 3dAllineate function. Following these transformations, functional images were smoothed using an $8.0 \mathrm{~mm}$ FWHM kernel and the $3 \mathrm{dmerge}$ function.

Three distinct GLMs were fit to functional data using the 3dDeconvolve function. One GLM (GLM1), designed to investigate choice difficulty effects using the contrast of catch and regular trials, included stick regressors for the onset of the first offer, and for the onset of the second offer, convolved with a canonical hemodynamic response (gamma) function. The decision difficulty contrast of regular and catch trials (comparing the magnitude of the response to the second offer onset) is reported in the statistics in Table 1, column 6. A second GLM (GLM2), not reported further here, used amplitude modulated "tent" functions to investigate trialwise modulation of signal by first offer SV in a wholebrain analyses to complement our core ROI-based analyses. A third GLM
(GLM3), used as the first stage of our core analyses, included regressors of no interest only. Residuals of GLM3 were used in most of our analyses to test for SV encoding, and derived statistics are reported in Figures 4-8 and Table 1, columns 3-5. All GLMs included six motion regressors, polynomial regressors suited to run duration for low-frequency drift, and a gamma-convolved stick function associated with the infrequent onset of a brief, $5 \mathrm{~s}$ reminder menu listing all load levels in their associated color labels. In addition to motion regression, we censored all frames with mean displacement $\geq 0.3 \mathrm{~mm}$ before parameter estimation to minimize the effects of high-motion transients (Siegel et al., 2014).

After fitting GLMs for individual subjects, we either contrasted average parameter estimates for regular and catch trials (GLM1) to test for decision difficulty effects, or fit linear models to test predictors of average BOLD signal residuals of GLM3 from a set of a priori ROIs to test for SV encoding. We used ROI-based analyses to maximize statistical power while protecting against false-positives, and because of strong a priori hypotheses about SV encoding in a putatively domain-general valuation network of 11 ROIs identified in two meta-analyses (Levy and Glimcher, 2012; Bartra et al., 2013). We contrasted these ROIs with 16 ROIs implicated in encoding SV in two recent studies of cognitive effort-based decision-making (Massar et al., 2015; Chong et al., 2017). To test for difficulty effects, we averaged parameter estimates from GLM1, estimated separately for difficult decision trials $(|\gamma| \leq 0.6)$, and easy catch trials $(|\gamma|=1.0)$, across all voxels lying within $6 \mathrm{~mm}$ radius spheres centered on loci of interest identified by prior literature. Next, we $t$ tested the differences in average parameter values across trial types for each ROI, or set of ROIs. To test for SV encoding, we extracted residuals of GLM3, and fit a series of mixed-effects models to estimate linear relationships between decision variables (SV, amount, and load) and residual BOLD signal variation. In all cases, we used exact peak coordinates as reported, except in the case of the amygdala, since the amygdala were slightly anterior of the center of mass reported in (Chong et al., 2017). For those loci, we used the anatomical amygdala centers as defined by Eickhoff et al. (2005). We then conducted all statistical analyses on averaged sphere values, across trials and subjects.

In the key analysis, we tested whether residuals of GLM3 encoded first-offer SV across trials and subjects, by fitting hierarchical linear models of residual BOLD signals $\left(Y_{i j}\right)$ as predicted by $z$-scored SV $\left(S V_{i j}\right)$ on trial $i$, with trials nested within participants $j$ :

$$
\begin{gathered}
Y_{i j}=B_{0 j}+B_{1 j} S V_{i j}+\varepsilon_{i j}, \\
B_{0 j}=\alpha_{00}+u_{0 j}, \\
B_{1 j}=\alpha_{10}+u_{1 j} .
\end{gathered}
$$

Note that the model is fully random, allowing both the intercept $B_{0 j}$, and SV effect $B_{1 j}$ to vary across participants. At the second level of the model, the intercept and slope are given subject-specific intercepts $\alpha_{00}$, and $\alpha_{10}$, respectively. This and all subsequent mixed models were fully random except in cases where predictor variables did not improve model deviance sufficiently to justify the additional degrees of freedom. $\varepsilon_{i j}, u_{0 j}$, and $u_{1 j}$ are all error terms.

When testing whether SV predicted variation in individual ROIs, we used the untransformed residuals from GLM3, averaged across all voxels in a given ROI, across 6-8 s following first-offer onset. However, for fitting models involving the network-level response of sets of ROIs (e.g., all ROIs in the putative domain-general valuation network) we combined all ROIs into a single outcome measure $\left(Y_{i j}\right)$ by $z$-scoring mean residual BOLD signals at $6-8 \mathrm{~s}$, across trials, for each ROI, then averaging these $z$-scores across ROIs in the network set for each participant. This method was used to give each component ROI equal weighting in quantifying the mean, network/set-level response on a given trial. For all hierarchical models, we used the arm package for R v1.10-1 (http:// CRAN.R-project.org/package $=$ arm).

We also tested whether linear combinations of ROIs, as a group, predict first offer SV in a series of nested, hierarchical model comparisons to ask whether residuals from one set of ROIs explain variance in SV better than another set, and vice versa. Note that this is a more stringent test of SV encoding than showing that variance is shared between trial-by-trial 
Table 1. Effects of choice difficulty and first offer SV, amount, and load on BOLD signal in all a priori ROIs, grouped by study of origin

\begin{tabular}{|c|c|c|c|c|c|}
\hline Anatomical description & $\operatorname{MNI}(x, y, z)$ & $S V, B \times 10^{-2}(p)$ & Amount, $B \times 10^{-2}(p)$ & Load, $B \times 10^{-2}(p)$ & Regular vs Catch $t_{(20)}(p)$ \\
\hline All SV meta-analysis ROIs & & $4.2(<0.01)^{* *}$ & $3.9(<0.01)^{* *}$ & $-4.8(<0.01)^{* *}$ & $1.58(0.13)$ \\
\hline \multicolumn{6}{|l|}{ Levy and Glimcher, 2012} \\
\hline I vmPFC & $(-7,38,-11)$ & $2.2(<0.01)^{* *}$ & $2.1(<0.01)^{* *}$ & $-2.1(<0.01)^{* *}$ & $-1.38(0.18)$ \\
\hline rvmPFC & $(4,35,-12)$ & $2.2(<0.01)^{* *}$ & $1.8(<0.01)^{* *}$ & $-2.3(<0.01)^{* *}$ & $-1.22(0.24)$ \\
\hline \multicolumn{6}{|l|}{ Bartra et al., 2013} \\
\hline $\mathrm{PCC}$ & $(-4,-30,36)$ & $1.6(0.03)^{*}$ & $1.4(0.03)^{*}$ & $-1.6(0.02)^{*}$ & $0.36(0.72)$ \\
\hline $\mathrm{dACC}$ & $(-2,16,46)$ & $1.9(0.05)^{*}$ & $0.7(0.39)$ & $-2.4(<0.01)^{* *}$ & $3.70(<0.01)^{* *}$ \\
\hline $\mathrm{ACC}$ & $(-2,28,28)$ & $1.6(0.06)^{\bullet}$ & $1.4(0.07)^{\bullet}$ & $-2.2(<0.01)^{* *}$ & $1.69(0.11)$ \\
\hline IVS & $(-12,12,-6)$ & $3.8(0.02)^{*}$ & $2.5(<0.01)^{* *}$ & $-1.8(<0.01)^{* *}$ & $0.92(0.37)$ \\
\hline rVS & $(12,10,-6)$ & $4.1(0.05)^{*}$ & $2.8(<0.01)^{* *}$ & $-1.8(0.02)^{*}$ & $0.39(0.70)$ \\
\hline r vmPFC & $(2,46,-8)$ & $3.1(<0.01)^{* *}$ & $2.4(0.01)^{*}$ & $-3.2(<0.01)^{* *}$ & $-1.48(0.16)$ \\
\hline IAl & $(-30,22,-6)$ & $0.8(0.10)^{\bullet}$ & $0.5(0.26)$ & $-1.2(<0.01)^{* *}$ & $3.28(<0.01)^{* *}$ \\
\hline rAl & $(32,20,-6)$ & $0.6(0.16)$ & $0.5(0.18)$ & $-0.8(0.02)^{*}$ & $2.70(0.01)^{*}$ \\
\hline Brainstem & $(-2,-22,-12)$ & $0.9(0.30)$ & $1.1(0.19)$ & $-1.6(0.05)^{\bullet}$ & $2.97(<0.01)^{* *}$ \\
\hline \multicolumn{6}{|l|}{ Massar et al., 2015} \\
\hline Massar et al. ROls (Study 1) & & $2.6(0.05)^{\bullet}$ & $1.8(0.19)$ & $-3.7(0.02)^{*}$ & $3.50(<0.01)^{* *}$ \\
\hline r supr. gyr. & $(33,-52,32)$ & $0.4(0.38)$ & $0.1(0.81)$ & $-0.7(0.09)^{\bullet}$ & $2.83(0.01)^{*}$ \\
\hline I cingulate & $(-24,-49,36)$ & $0.4(0.24)$ & $0.1(0.63)$ & $-0.5(0.13)$ & $2.61(0.02)^{*}$ \\
\hline IITG & $(-58,-35,-22)$ & $1.1(0.08)^{*}$ & $0.5(0.25)$ & $-1.7(<0.01)^{* *}$ & $-1.78(0.09)^{\bullet}$ \\
\hline IIFG & $(-43,53,-4)$ & $0.4(0.61)$ & $0.4(0.67)$ & $-1.8(0.03)^{*}$ & $0.66(0.51)$ \\
\hline IIPL & $(-30,-43,43)$ & $0.5(0.35)$ & $0.3(0.48)$ & $-0.7(0.12)$ & $5.10(<0.01)^{* *}$ \\
\hline IIPL & $(-41,-55,46)$ & $-0.2(0.82)$ & $0.1(0.93)$ & $-0.9(0.22)$ & $3.42(<0.01)^{* *}$ \\
\hline r temp. pole & $(34,16,-26)$ & $3.1(0.03)^{* *}$ & $1.8(0.02)^{*}$ & $-1.4(0.08)^{\bullet}$ & $0.04(0.97)$ \\
\hline \multicolumn{6}{|l|}{ Chong et al., 2017} \\
\hline Chong et al. ROls (Study 2) & & $2.1(0.11)$ & $1.6(0.24)$ & $-3.5(0.05)^{*}$ & $3.41(<0.01)^{* *}$ \\
\hline I amygdala & $(-24,0,-22)$ & $2.2(0.08)^{\bullet}$ & $1.6(0.04)^{*}$ & $-1.7(0.04)^{*}$ & $-0.41(0.69)$ \\
\hline r amygdala & $(24,0,-22)$ & $2.8(0.03)^{*}$ & $2.1(0.02)^{*}$ & $-1.4(0.13)$ & $-0.15(0.88)$ \\
\hline IIPS & $(-36,-44,38)$ & $0.4(0.50)$ & $0.1(0.90)$ & $-0.8(0.12)$ & $6.04(<0.01)^{* *}$ \\
\hline $\mathrm{dmPFC}$ & $(-4,22,44)$ & $1.1(0.12)$ & $0.4(0.56)$ & $-1.9(<0.01)^{* *}$ & $2.61(0.02)^{*}$ \\
\hline rIPS & $(24,-60,42)$ & $0.6(0.25)$ & $0.3(0.56)$ & $-1.0(0.04)^{*}$ & $4.95(<0.01)^{* *}$ \\
\hline rAl & $(34,22,2)$ & $0.2(0.75)$ & $0.2(0.65)$ & $-0.4(0.30)$ & $3.78(<0.01)^{* *}$ \\
\hline I dIPFC & $(-44,26,26)$ & $0.9(0.23)$ & $0.8(0.18)$ & $-0.6(0.36)$ & $3.29(<0.01)^{* *}$ \\
\hline rdIPFC & $(44,36,30)$ & $0.1(0.95)$ & $-0.4(0.55)$ & $-1.3(0.07)^{\bullet}$ & $1.68(0.11)$ \\
\hline l insula & $(-28,22,4)$ & $0.5(0.17)$ & $0.3(0.24)$ & $-0.5(0.11)$ & $3.08(<0.01)^{* *}$ \\
\hline
\end{tabular}

Column 2, MNI coordinates for each ROI. Columns 3-5, Effect estimates and corresponding $p$ values for activity in a priori networks and individual R0Is predicting mean residual activity $6-8 \mathrm{~s}$ after first offer onset, after regressing out motion and other predictors of non-interest. Column 3 describes first offer SV as a predictor of residuals. Columns 4 and 5 provide relationships with first offer amount and load simultaneously estimated in a hierarchical multiple regression. Column $6, t$ test contrasting the canonical hemodynamic response on regular versus catch trials. Cells are starred according to their $p$ value: ${ }^{* *} p<0.01,{ }^{*} 0.01<p<0.05$, and ${ }^{\bullet} 0.05<p<0.10$. Network-level effects at the top of each section were estimated from the mean response at $6-8 \mathrm{~s}, z$-scored across trials, then averaged across ROls in each network.

SV and BOLD signals in a single network. Showing that one set of a priori regions predicts SV while controlling for variance in other regions demonstrates both more robust SV encoding relative to other networks, and implies greater specificity in encoding SV versus more general factors like arousal, which may evoke activity across multiple networks. For this analysis, we fit the following models including untransformed ROI residuals (of GLM3) from set $A$ alone (ROIA) in the first step, then included ROI residuals from set $B(R O I B)$ in the second step. $n_{a}$ is the count of ROIs in set $A$, etc.

$$
\begin{gathered}
S V_{i j}=B_{0 j}+\sum_{a=1}^{n_{a}} B_{a} R O I A_{i}+\varepsilon_{i j}, \\
S V_{i j}=B_{0 j}+\sum_{a=1}^{n_{a}} B_{a} R O I A_{i}+\sum_{b=1}^{n_{b}} B_{b} R O I B_{i}+\varepsilon_{i j}, \\
B_{0 j}=\alpha_{00}+u_{0 j} .
\end{gathered}
$$

After estimating each model, we compared model fit according to $\chi^{2}$ distributed deviance, to ask whether the additional regressors explained significantly more variance than the nested model.

Mirroring our analysis of trialwise SV encoding, we also tested whether amount and load are jointly predictive of GLM3 residuals, by fitting additional hierarchical linear models that replaced SV with these two terms. Note, however, that we did not model random effects for amount and load (only the intercept term was both fixed and random) as they were fixed across participants, and because including random effects for these predictors did not explain sufficient variance to justify the additional degrees of freedom according to a nested model comparison.

$$
Y_{i j}=B_{0 j}+B_{1} \text { Amount }_{i}+B_{2} \text { Load }_{i}+\varepsilon_{i j} \text {. }
$$

Additionally, we tested whether a measure of mean willingness to accept a high effort-cost, high reward-benefit offer predicted BOLD signal residuals in putative valuation ROIs as a main effect and in interaction with first offer amount and load. Because mean willingness to accept a high-cost, high-benefit offer indexes trait cognitive motivation (Westbrook et al., 2013), interactions with load and amount would imply that individuals with higher cognitive motivation were less sensitive to offer load or more sensitive to offer amounts, respectively. To test this hypothesis, we used area under the discounting curve (AUC; equivalent here to the average of SVs across all high-load, amount pairings) for each individual. Because we were interested in using AUC as a trait measure of cognitive motivation, we averaged together AUCs measured on three separate occasions (two prior visits and the imaging visit), to estimate stable discounting tendencies. For testing whether AUC, at the subject level, predicts GLM3 residuals as a main effect, and in interactions with amount and load, we fit the following model. Here, amount and load are treated as fixed effects, whereas AUC was a predictor of those effects at the participant level of the model.

$$
Y_{i j}=B_{0 j}+B_{1 j} \text { Amount }_{i}+B_{2 j} \text { Load }_{i}+\varepsilon_{i j}
$$




$$
\begin{gathered}
B_{0 j}=\alpha_{00}+\alpha_{01} A U C_{j}+u_{0 j}, \\
B_{1 j}=\alpha_{10}+\alpha_{11} A U C_{j}, \\
B_{2 j}=\alpha_{20}+\alpha_{21} A U C_{j} .
\end{gathered}
$$

The logic of this analysis is that if either of the cross-level interaction terms $\alpha_{11}$ or $\alpha_{21}$ were significant predictors of BOLD residuals, this would indicate that SV representations were modulated by individual differences in participants' trait cognitive motivation (indexed by AUC) via differential sensitivity to amount benefits or load costs, respectively.

To investigate whether SV representations varied with trialwise motivation, we tested for relationships between choice patterns and offer biasing (the proximity parameter, $\gamma$ ). Specifically, we asked whether BOLD signals in putative valuation ROIs were higher or lower on trials in which participants ultimately selected the hard task offer, even when we offered more for the easy task (relative to the SV of hard task), or vice versa. Thus, we tested whether choice and second offer bias predicted GLM3 residuals $6-8 \mathrm{~s}$ after first offer onset, fitting a model with trials, $i$, 3 vmPFC ROIs, $k$, nested within participants, $j$.

$$
\begin{gathered}
Y_{i k j}=B_{0 k j}+B_{1} \text { ChcBias }_{i}+\varepsilon_{i k j}, \\
B_{0 k j}=\alpha_{00 j}+\varepsilon_{0 k j}, \\
\alpha_{00 j}=\nu_{000}+u_{00 j},
\end{gathered}
$$

The predictor ChcBias was coded to incorporate two factors: (1) whether the low or high-demand option was chosen; and (2) whether the amount for the low-demand option was above $(\gamma>0)$ or below the SV of the high-demand option $(\gamma<0)$, thus biasing choice toward the low ("low-load biased") or high-demand option ("high-load biased"), respectively. We coded the predictor to indicate whether participants chose in accordance with, or against these offer biases. The regressor took the following values, mapping increasing motivation to select the high-cost, high-benefit option on a given trial. For low demand chosen, high-load biased trials (choices implying low momentary cognitive motivation), ChcBias $=-1.5$, for low demand chosen, low-load biased trials, ChcBias $=-0.5$, for high demand chosen, high-load biased trials, Ch$c$ Bias $=0.5$, and for high demand chosen, low-load biased trials (choices implying high momentary cognitive motivation), ChcBias $=1.5$. This coding scheme thus emphasizes the effect of higher state motivation reflected to select the high-load offer, particularly when choice was biased against this offer. In this analysis, if the ChcBias term positively predicted $\mathrm{SV}$, this would indicate that more positive SV representations on a given trial were related to higher implied motivation.

We also considered the possibility that the effect of load on SV representations might depend on performance of the prior $N$-back task (e.g., the possibility that load-related cost encoding might be stronger for those who performed worse on higher load levels). To investigate whether level-specific $N$-back performance $\left(d_{i j}^{\prime}\right)$ predicted BOLD signal residuals 6-8 s after first offer onset $\left(Y_{i j}\right)$ either as a main effect or in interaction with load on trial $i$ for participant $j$, the following multilevel models were estimated in the dACC, and the bilateral anterior insula (AI).

$$
\begin{gathered}
Y_{i j}=B_{0 j}+B_{1} \text { Amount }_{i}+B_{2} \text { Load }_{i}+B_{3} d_{i j}^{\prime}+B_{4 j}\left(d_{i j}^{\prime} \times \operatorname{Load}_{i}\right)+\varepsilon_{i j}, \\
B_{0 j}=\alpha_{00}+u_{0 j}, \\
B_{4 j}=\alpha_{40}+u_{4 j} .
\end{gathered}
$$

Finally, to test whether SV predicted BOLD signal 6-8 s after first offer onset, simply controlling for performance at that task load, we examined regression weights for the SV term in the following model.

$$
\begin{gathered}
Y_{i j}=B_{0 j}+B_{1 j} S V_{i j}+B_{2} d_{i j}^{\prime}+\varepsilon_{i j}, \\
B_{0 j}=\alpha_{00}+u_{0 j}, \\
B_{1 j}=\alpha_{10}+u_{1 j} .
\end{gathered}
$$

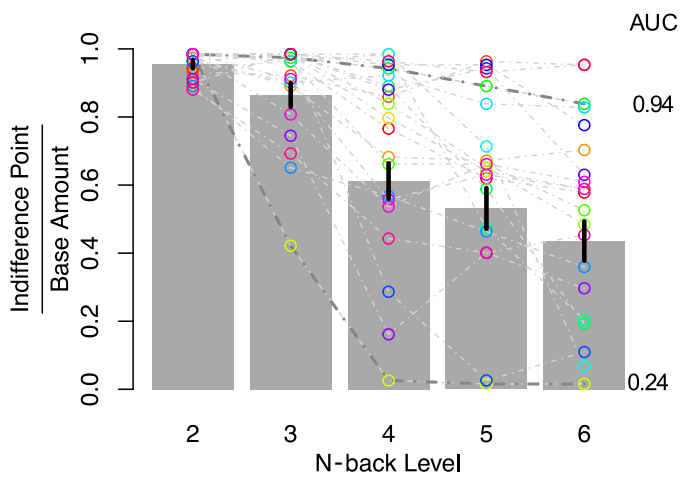

Figure 1. Decreasing indifference points (here normalized by, and averaged over base amounts) reflect rising subjective costs as $\mathrm{N}$-back level increases. Gray bars and black lines reflect group means \pm SEM. Gray dashed lines show individual participants' discounting curves, illustrating clear individual differences. AUC values provided for two example participants.

\section{Results}

\section{Reward is discounted subjectively by cognitive load}

In the COGED paradigm, participants decide whether to perform $\mathrm{N}$-back working memory task levels that vary in cognitive load for differing amounts of money (Kirchner, 1958; Braver et al., 1997). Prior work has demonstrated that the $N$-back is perceived as effortful, and self-reported effort increases systematically with cognitive load (Westbrook et al., 2013; Hopstaken et al., 2015). Critically, subjective effort costs are measured by the extent to which cash offers are discounted for each load level ( $N$; Westbrook et al., 2013). In the present study, outside the scanner, participants first experienced all $N$-back levels, then made repeated decisions between performing a high-load $N$-back level $(N=2-6)$ for one of three base offer amounts $(\$ 2, \$ 3$, or $\$ 4)$ or instead performing the low-load 1-back condition for a smaller, variable amount. Low-load (1-back) offers were iterated in a staircase fashion, until an indifference point was reached. Indifference points give the SV: the perceived worth of monetary offers, discounted by costs of each higher $N$-back load relative to the low-load baseline. Replicating prior findings (Westbrook et al., 2013), offers were discounted at all (high) load levels, and moreover an ANOVA of indifference points normalized by base offer amounts revealed that participants discounted offers proportionally more for larger loads $\left(F_{(1,20)}=49.3 ; p=8.2 \times 10^{-7} ; \eta^{2}\right.$ $=0.68$ ), indicating increasing effort costs (Fig. 1). Additionally, participants discounted proportionally less for larger amounts $\left(F_{(1,20)}=11.6 ; p=0.0028 ; \eta^{2}=0.06\right)$ and an interaction $\left(F_{(1,20)}\right.$ $\left.=6.87 ; p=0.016 ; \eta^{2}=0.01\right)$ indicated that amount effects were largest for higher load levels, though the amount and interaction effect sizes were small compared with the load effect. Importantly, there were also strong individual differences in the degree of discounting (Fig. 1). Given theoretical uncertainty regarding the form of the discount function (Hartmann et al., 2013; Chong et al., 2017), we quantified individual differences with AUC (Fig. 1 ), a measure that prior work has shown to be psychometrically optimal for individual difference analyses (Myerson et al., 2001). Higher AUC indicates that a participant is more willing to expend cognitive effort for reward, on average.

Participants may discount high-effort tasks because they anticipate worse performance as task load increases. However, it is unlikely that discounting reflects performance alone. First, participants were instructed that they would be paid for completing a task, even for poor performance. Second, although poor per- 
formance predicts steeper discounting, there is considerable discounting variance not explained by performance. For example, even controlling for $N$-back performance $\left(d^{\prime} ; B=9.83 \times 10^{-2}\right.$, $p=0.019)$, load significantly $\left(B=-8.16 \times 10^{-2}, p=9.1 \times\right.$ $10^{-10}$ ) predicted SV in a hierarchical multiple regression (load levels nested within participants). Furthermore, indexed by $d^{\prime}$, steep discounters (below-median AUC) performed the $N$-back as well as shallow discounters (Wilcoxon $p=0.62$ ), and numerically better at high-load levels (though not reliably: $p$ values $\geq 0.16$ ). Hence, although declining performance with higher load may contribute to discounting, it does not satisfactorily explain load effects or individual differences.

In the fMRI scanner, participants again decided between a base offer $(\$ 2, \$ 3$, or $\$ 4)$ to perform the high-load $N$-back $(N=$ 2-6), and a variable amount for the low-load, but this time with the low-load offer systematically adjusted with respect to participants' own indifference points, to control decision difficulty and balance choice bias. Specifically, with $\gamma$ referring to the fractional difference between the indifference offer and the bounds of $\$ 0$ or the base amounts $(\$ 2, \$ 3$, or $\$ 4)$, participants decided between offers in which the low-load amount was slightly above indifference ( $\gamma=0.2,0.6)$, biasing low-load choices (low-load biased), or slightly below indifference $(\gamma=-0.1,-0.4)$, biasing high-load choices (high-load biased trials). We also included catch trials, in which we offered equal amounts $(\gamma=1.0)$ for the 1-back and high-load task, strongly biasing low-load choices ("low-load catch") or instead $\$ 0(\gamma=-1.0)$, strongly biasing high-load choices ("high-load catch"). A key advantage of this design is that decision difficulty, high-load versus low-load preference, and firstoffer SV were all orthogonalized across trials.

Bias reliably $\left(F_{(1,20)}=341 ; p=4.9 \times 10^{-14}\right)$ influenced the probability of selecting the high-load offer on a given trial. Excluding catch trials, there was a reliable preference for high-load offers on high-load biased trials (choice probability $>0.5 ; \mathrm{t}_{20}=$ 5.12; $\left.p=5.3 \times 10^{-5}\right)$, and a for low-load offers on low-load biased trials $\left(\gamma>0\right.$; choice probability $<0.5 ; t_{(20)}=2.31 ; p=$ 0.031; Fig. 2A).

Choice reaction times provide further evidence that our discounting procedure identified indifference points accurately: participants responded more slowly on trials closer to indifference, consistent with increasing decision difficulty. On "probias" trials, in which participants' choices were consistent with offer biases, median reaction times were slower on more difficult decision trials $(|\gamma|<1.0)$ relative to catch trials ( $\gamma=-1.0$ or 1.0; $t_{(20)}=7.51, p=3.0 \times 10^{-7}$; Fig. 2B). Furthermore, when decisions went against offer biases ("anti-bias" trials), reaction times were significantly slower than on pro-bias trials $\left(t_{(20)}=7.03, p=8.1 \times\right.$ $\left.10^{-7}\right)$. Such a pattern is consistent with a response conflict account of decision difficulty (Yarkoni et al., 2005; Botvinick, 2007; Pochon et al., 2008), as conflict would be highest, on average, when deciding against typically preferred alternatives.

\section{SV encoding}

Our central question was whether a domain-general valuation network encoded cognitive effort-discounted SV. To test this, we asked whether BOLD response reliably tracked the SV (indifference points) of offers to repeat $N$-back tasks for money. Specifically, participants were instructed to consider the value of single high-load $N$-back options $(N=2-6)$ paired with single base amounts $(\$ 2, \$ 3$, or $\$ 4$ ), presented in isolation for 6 s (i.e., 1 of 15

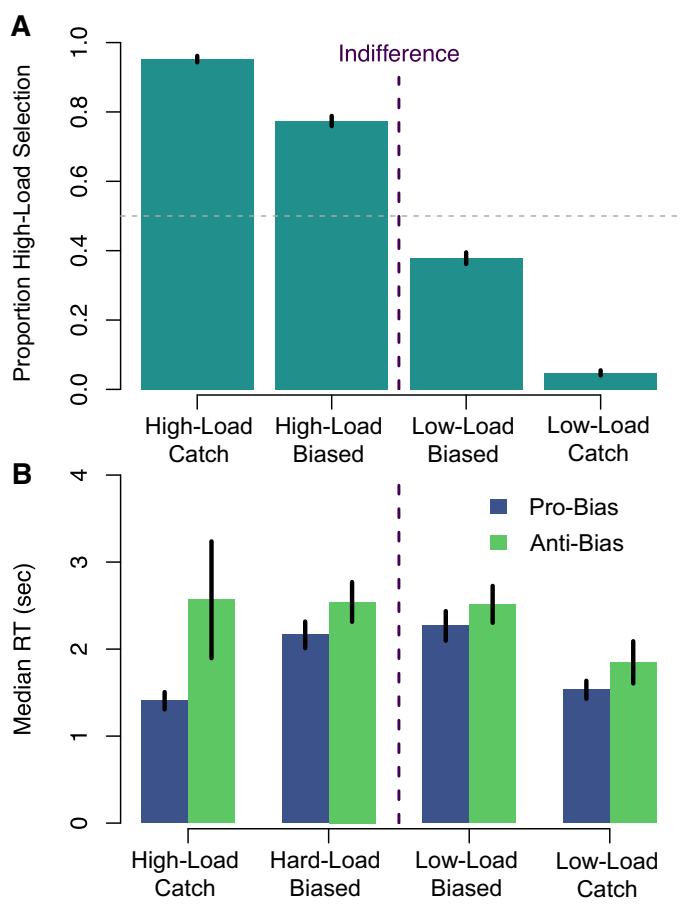

Figure 2. $\quad A$, Proportion of high-demand options selected as a function of offer biasing, and $\mathrm{N}$-back level. $\boldsymbol{B}$, Median reaction times as a function of proximity and whether choices went with (pro-bias) or against (anti-bias) offer biasing (High-load: $\gamma<0$; Low-load: $\gamma>0$ ).

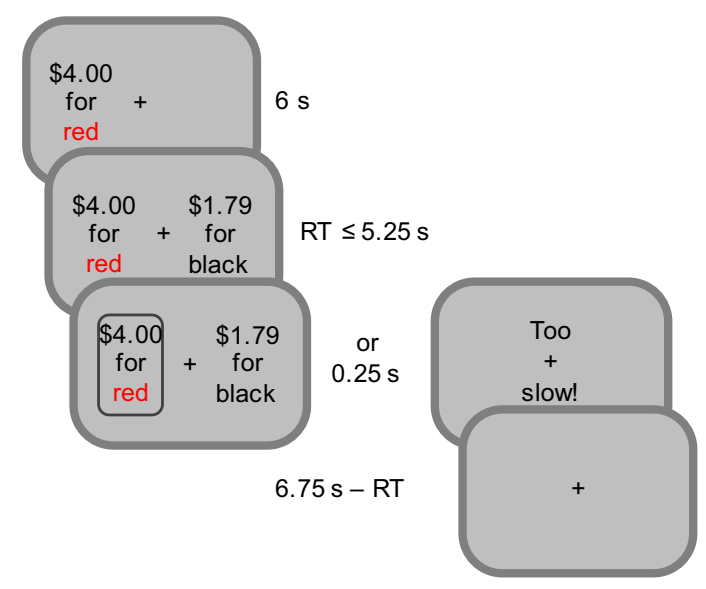

Figure 3. Imaging trials begin with a high-amount, high-load offer (load is indicated by a color label, e.g., red for 2-back). After $6 \mathrm{~s}$ the 1-back (black task) offer is presented. Participants have $5.25 \mathrm{~s}$ to respond. After response is indicated briefly, a fixation cross is presented until the end of the trial.

high-load offers were presented first; Fig. 3). Consequently, brain activity $6-8 \mathrm{~s}$ after first offer onset was regarded as reflecting single offer valuation, isolated from other decision processes, accounting for hemodynamic lag (Miezin et al., 2000). We tested whether activity during this "valuation period" tracked trialby-trial, first-offer SV within a set of 11 ROIs from prior metaanalyses identifying the putatively domain-general core valuation network (Fig. 4A, yellow), which includes the vmPFC, VS, AI, posterior cingulate cortex (PCC), and dACC (Levy and Glimcher, 2012; Bartra et al., 2013). The focus on a priori ROIs was motivated by strong prior beliefs about regions encoding $\mathrm{SV}$, and the desire to maximize statistical power (for completeness, we also conducted a whole-brain analysis although this did not identify any regions outside of 


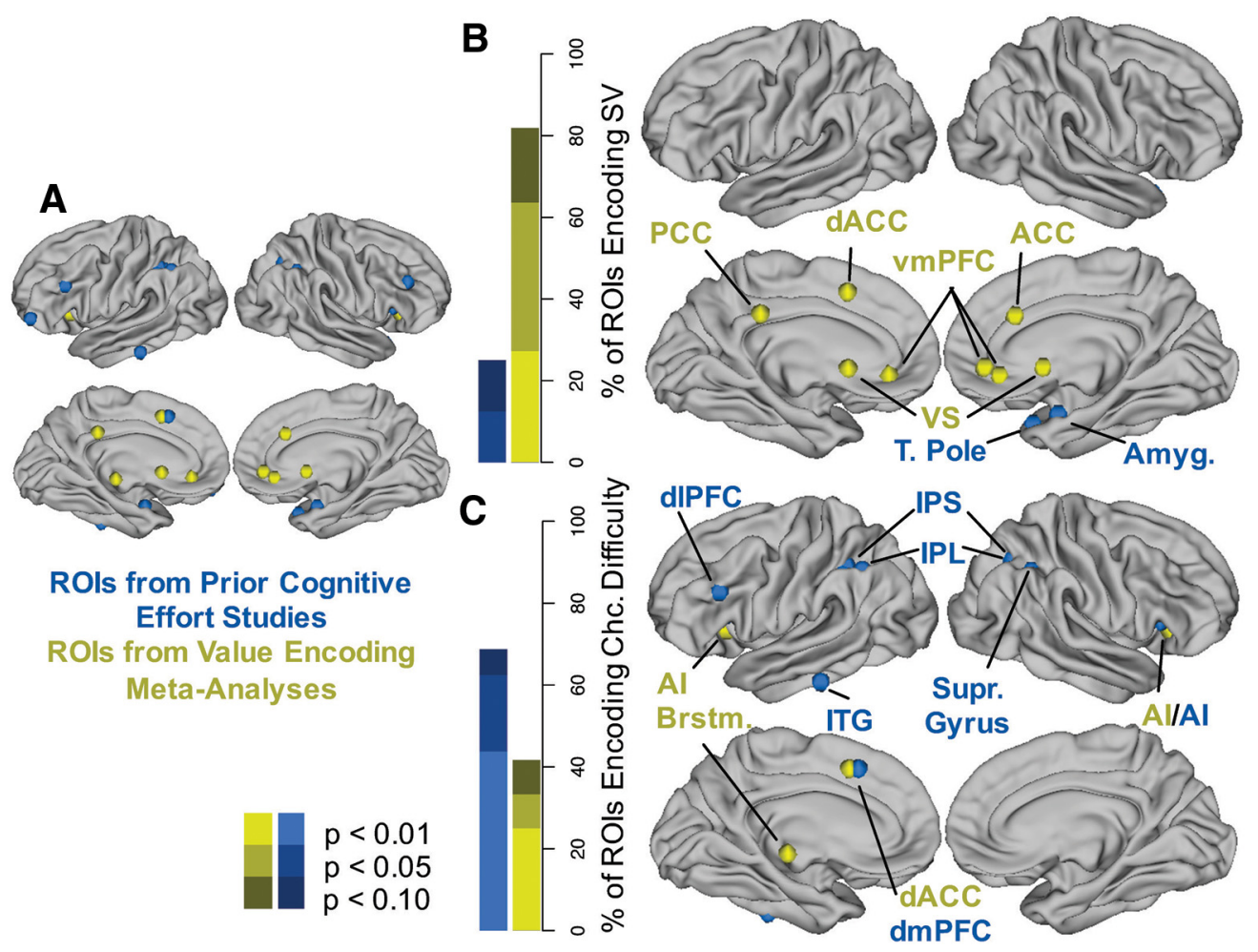

Figure 4. A, Six-millimeter-radius spheres centered at all a priori ROls. Colors indicate origination from either of two prior meta-analyses of domain-general SV encoding (yellow, Levy and Glimcher, 2012; Bartra et al., 2013) or two recent studies on SV encoding during cognitive effort decision-making (blue, Massar et al., 2015; Chong et al., 2017). Note that the amygdala is projected to the surface for display purposes only. B, Percentage of ROls from each set tracking SV $6-8 \mathrm{~s}$ after first offer onset by $p$ value. Map shows ROls reliably tracking SV at $p<0.05$. C, Percentage of ROIs from each set with reliably more activity on difficult, regular versus easy catch trials by $p$ value. Map shows ROls with a reliable difficulty contrast at $p<0.05$. T Pole, Temporal pole; Amyg, amygdala; Supr Gyrus, supramarginal gyrus; ITG, inferior temporal gyrus; Brstm, brainstem.

our candidate ROIs). We contrasted the SV encoding in this meta-analysis defined core valuation network with sets of ROIs (16 in total) identified in two recent studies specifically focused on decision-making about cognitive effort (Fig. 4A, blue; Massar et al., 2015; Chong et al., 2017). Note that in addition to testing whether individual ROIs tracked SV from trial-to-trial, we combined ROIs as a set, giving each ROI equal weight by $z$-scoring each ROI's response at $6-8 \mathrm{~s}$, then averaging to obtain network-level responses.

Our data indicate clearly that the meta-analysis core valuation network of regions positively and reliably tracked firstoffer SV in the valuation period, $6-8 \mathrm{~s}$ following the first offer onset $\left(B=4.09 \times 10^{-2} ; p=2.6 \times 10^{-4}\right)$. Moreover, almost all individual ROIs in this set showed some evidence of tracking first offer SV (at $p<0.05$ ) including the bilateral vmPFC, VS, PCC, ACC, and dACC (Fig. 4B; Table 1, column 3). These results are consistent with the hypothesis that a core valuation network encodes SV, discounted by cognitive effort costs. Furthermore, they extend the notion of domain-generality from delay, risk, and physical effort costs (Levy and Glimcher, 2012; Bartra et al., 2013) to the domain of cognitive effort. Among individual ROIs, only the brainstem and right AI showed no evidence of encoding effort-discounted SV, whereas the left AI showed only trend-level evidence, despite these regions reliably encoding SV in other cost domains (Bartra et al., 2013).

By contrast, SV encoding was unreliable in the set of ROIs identified in the prior cognitive effort studies. The set of ROIs from one study (Study 1; Massar et al., 2015) encoded SV at trend-level $\left(B=2.57 \times 10^{-2} ; p=0.050\right)$, whereas the set identified in by the other study (Study 2; Chong et al., 2017) did not
$\left(B=2.13 \times 10^{-2} ; p=0.11\right)$. Moreover, unlike the meta-analysis set, most individual ROIs from either study did not reliably track first-offer SV. Notable exceptions to this pattern include the right temporal pole from Study 1 and right amygdala from Study 2 (the left amygdala also encoded trial wise SV at trend-level). The amygdala is notable as a region that has been previously implicated in encoding SV during cognitive, relative to physical effortbased decision-making (Chong et al., 2017) and also supporting cognitive effort-based decision-making in rats (Hosking et al., 2014). Despite these exceptions, the broader pattern did not replicate the findings from the two prior cognitive effort studies: in neither the IPS, parietal lobule (IPL), nor the lateral PFC loci did activity reliably track first offer SV.

Direct comparisons between sets of ROIs reveal that the metaanalysis core valuation network not only tracks trialwise SV with greater reliability than ROIs identified in the two prior cognitive effort studies, but also that activity in these ROIs is modulated more strongly by, and in turn explains more trial-by-trial variance in, first offer SV. First, as a group, valuation network regression weights (Table 1, column 3) are reliably larger across individual ROIs than those from the two prior cognitive effort studies $\left(t_{(19)}=2.78 ; p=0.012\right)$. Second, in hierarchical, nested model comparisons of pro-bias trials, valuation network ROIs explained variance in first-offer SV above and beyond that explained by the ROIs from the prior effort studies. For example, after controlling for activity $6-8 \mathrm{~s}$ after first offer onset in all ROIs from Study 1 and Study 2, adding activity from the left $\left(\chi_{1}^{2}=10.4\right.$, $p=0.0013)$ or right $\left(\chi_{1}^{2}=8.52, p=0.0035\right) \mathrm{VS}$, or left $\left(\chi_{1}^{2}=4.00\right.$, $p=0.046)$ or right $\left(\chi_{1}^{2}=2.86, p=0.091\right)$ vmPFC improved $\chi^{2}$ 
distributed (Gelman and Hill, 2007) model deviance, indicating that the valuation network ROIs explain reliably more variance in first-offer SV across trials. When adding all 11 valuation ROIs at once, model deviance also improved at trend-level $\left(\chi_{11}^{2}=18.01\right.$, $p=0.081$ ) above a model including all ROIs from Study 1 and Study 2. By contrast, adding Study 1 and Study 2 ROIs to a base model containing valuation ROIs did not improve model deviance $\left(\chi_{16}^{2}=12.25, p=0.73\right)$.

\section{Encoding of decision difficulty}

Relatively weak SV encoding among ROIs from the prior cognitive effort studies suggests that they were previously implicated because SV encoding is only apparent under certain conditions, or that the ROIs they identified are primarily responsive to factors correlated with SV, rather than SV per se. One possibility is decision difficulty. Correlation between SV and difficulty might occur, for example, if participants typically prefer high-effort, high-reward alternatives (such alternatives have higher SV on most trials), and the decision only becomes difficult on infrequent trials when the SV of the high-effort option is low and differences in SV between offers are small. This is a particularly likely explanation in the case of Study 2, in which participants mostly preferred the high-effort option. Moreover, Study 2 regressed BOLD signal on the difference in the SV of a chosen offer and that of a fixed baseline. Thus, the SV difference regressor used in that study should negatively covary with difficulty. Study 1 , by contrast, orthogonalized the value of the high-cost offer with respect to the low-cost offer, and included reaction time as a covariate to control for choice conflict. Also, selection rates of the high- and low-effort options were balanced. Nevertheless, both studies examined BOLD signal while participants compared two offers, rather than evaluating a single offer, leaving open the possibility that ROIs identified in the prior studies tracked subtle aspects of SV that were correlated with decision difficulty. Also, in both cases, it is possible that SV regressors interacted with difficulty and were thus most predictive of BOLD signal when choice difficulty was high. Perhaps the ROIs identified in these prior studies are mechanisms recruited specifically on difficult trials involving close offer comparison.

To examine whether ROIs from the prior cognitive effort studies were indeed more sensitive to decision difficulty, we tested whether they were more sensitive to differences in offer SV. Specifically, we contrasted hemodynamic response functions, time-locked to second offer onset, between difficult trials, when the differences in SV of were small $(|\gamma|<0.6)$, and easy catch trials, when differences in SV were large $(\gamma=-1.0$ or 1.0). In a reversal of the SV encoding analysis, sets of ROIs from the prior cognitive effort studies were robustly sensitive to this difficulty contrast (Study 1: $t_{(20)}=3.50, p=0.0022$; Study 2: $t_{(20)}=3.41$, $p=0.0028)$, whereas the meta-analysis valuation network was not $\left(t_{(20)}=1.58, p=0.13\right)$. The pattern of results across sets of ROIs suggests a double dissociation, which is buttressed by a strikingly consistent pattern at the individual ROI level: individual ROIs were either more reliably active on difficulty trials or reliably tracked SV, but not both (Table 1; Fig. 4B,C). Sole exceptions to this pattern were the dACC and trend-level results in the inferior temporal gyrus and left AI. As noted, ROIs sensitive to the difficulty contrast comprise regions more typically associated with cognitive control, working memory, and cost-benefit evidence accumulation (Braver et al., 1997; Dosenbach et al., 2006; Kouneiher et al., 2009; Basten et al., 2010), including the bilateral IPS, the dACC and pre-supplementary motor area, and dlPFC. As above, for completeness, we examined the decision difficulty contrast across the whole brain; the results recapitulated the ROI analysis: a network of regions including the dACC, dIPFC, and IPS were more active on difficult versus easy trials, whereas valuation network regions were not differentially active across trial types. This pattern of results indicates a double dissociation, in which a domain-general core valuation network tracks first-offer SV significantly better than frontoparietal regions implicated in prior cognitive effort studies, whereas conversely these latter frontoparietal regions are more sensitive to decision difficulty than those involved with domain-general valuation.

\section{Costs and benefits are jointly encoded in the valuation network}

For a region to track SV, it should encode both offer benefits and costs, and with opposing signs. Surprisingly few SV encoding studies decompose these sources of variance, however, which leaves open the possibility that BOLD signal correlating with SV may be primarily tracking costs or benefits alone. Thus, we tested whether trial wise first-offer amount (benefits) and $N$-back load (costs) jointly and independently predicted mean valuation period (at 6-8 s) activity in a priori ROIs, using hierarchical linear models. We found that the meta-analysis valuation network covaried both positively with amount (controlling for load; $B=3.91 \times 10^{-2}$; $p=$ 0.0035 ), and negatively with load (controlling for amount; $B=$ $-4.80 \times 10^{-2} ; p=3.6 \times 10^{-4}$; Table 1). This was also true, moreover, among most individual ROIs within this network. For example, bilateral vmPFC and VS activity both reliably increased with higher-offer amounts and decreased with increasing task loads (Fig. 5). This result confirms that the putatively domain-general valuation network not only correlates with SV, but independently tracks both cognitive costs and benefits. By contrast, and mirroring our SV analysis, we did not find encoding of both dimensions in the sets of regions implicated by the prior cognitive effort studies. In both cases, we found evidence that these networks encoded first-offer load (Study 1: $B=-3.69 \times 10^{-2}, p=0.023 ;$ Study $2: B=-3.41 \times 10^{-2}$, $p=0.048$ ), but not first offer amount (Study $1: B=1.79 \times 10^{-2}, p=$ 0.19 ; Study 2: $B=1.58 \times 10^{-2}, p=0.24$ ). Relative insensitivity to first offer amount helps explain why these ROIs did not reliably track first-offer SV.

There were some individual ROIs within the meta-analysis valuation network, however, for which our data only support the encoding of single dimensions. For example, load reliably (and negatively) predicted activity in the AACC and the bilateral AI (all $p$ values $\leq$ 0.02 ), but reward did not (all $p$ values $\geq 0.18$ ). These findings leave open the possibility that certain ROIs tracking $\mathrm{SV}$ in other studies may have reflected encoding of specific dimensions, rather than SV per se. There may be methodological reasons, however, why these ROIs do not track first-offer amount, (e.g., low power) so negative results in individual ROIs should be interpreted with caution.

\section{Individual ROIs encoding value}

Although the set of meta-analysis valuation ROIs showed clear evidence of SV encoding at the network level, it is also useful to know whether individual ROIs encode SV. Although most ROIs showed some evidence of encoding SV (Table 1), none of the individual 11 valuation ROIs survived Benjamini-Hochberg-Yekutieli FDR correction (Benjamini and Hochberg, 1995). By contrast, both the left $(-7,38,-1)$ and right vmPFC $(4,35,-12)$ and the left VS $(-12,12,-6)$ survived multiple-comparisons correction for reliably encoding amount and load, indicating robust evidence that the vmPFC and VS encode both key dimensions of SV. Beyond these three ROIs, the right VS $(12,10,-6)$, dACC $(-2,16,46)$, ACC $(-2,28,28)$, and left AI $(-30,22,-6)$ also sur- 

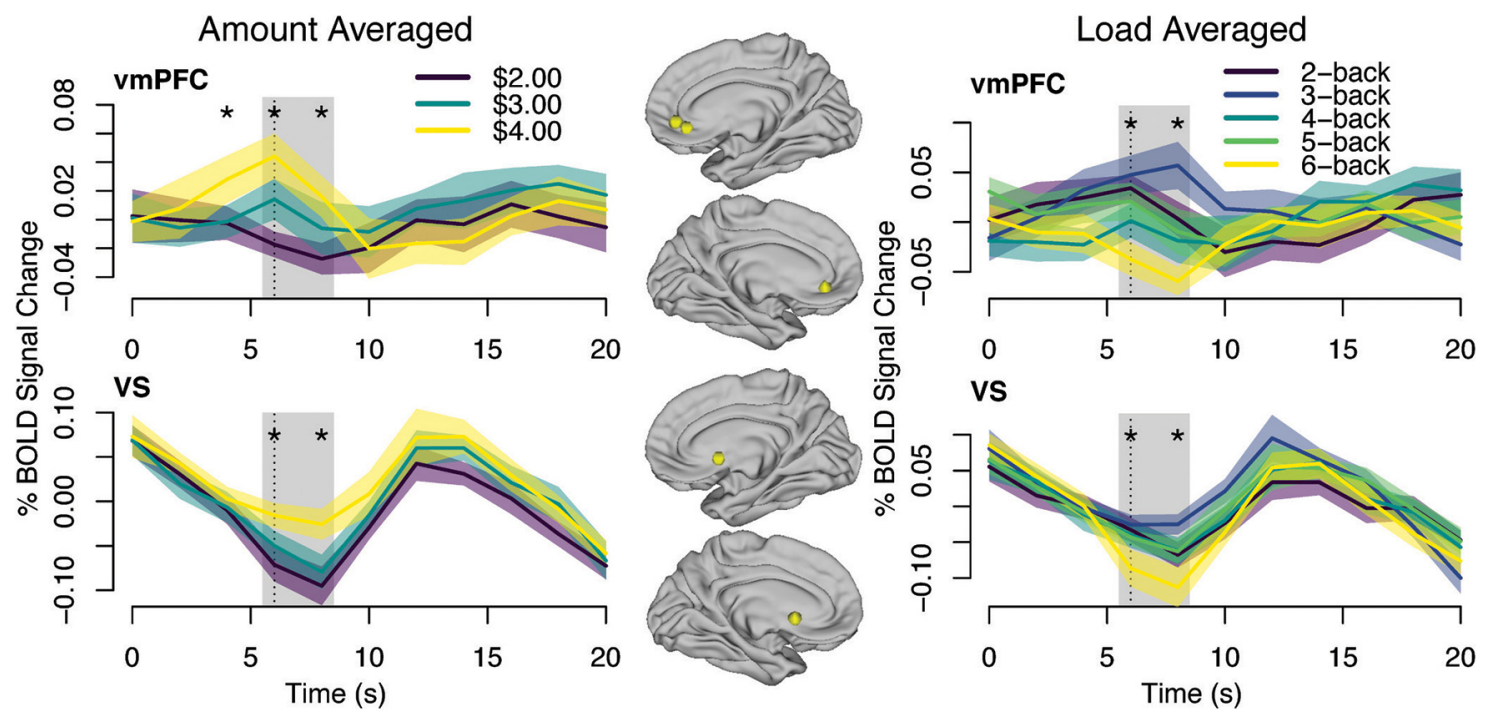

Figure 5. Residual time courses in a priori ROIs, averaged by first offer amount or load. Error bands reflect \pm SEM across participants. Reliability of amount and load effects in separate hierarchical multiple regression (trials and ROIs, nested within participants) at each time point indicated by ${ }^{*} p<0.05$. Gray region highlights $6-8 \mathrm{~s}$ after first offer onset; vertical dashed line indicates second offer onset.

vived FDR correction for encoding load but not amount. Outside of the meta-analysis valuation ROIs, neither any of the seven ROIs from Study 1, nor any of the nine ROIs from Study 2 survived FDR correction for encoding either amount or load.

\section{Trait subjectivity in value encoding}

Beyond objective dimensions like reward amount and task load, SV further implies subjectivity in how those dimensions are experienced. Indeed, participants varied considerably in their willingness to exert effort for reward across load and reward levels (Fig. 1). Subjectivity may partly reflect stable, trait experience. To test for trait subjectivity in the experience of offers, we used AUC, a measure of participants' overall tendency to accept an offer to perform a high-load $\mathrm{N}$-back for reward. Prior work has shown that COGED AUC predicts personality traits and individual differences in delay discounting, cognitive aging, and negative schizophrenia symptoms (Westbrook et al., 2013; Culbreth et al., 2016), supporting its use as a trait measure. Furthermore, we took advantage of the fact that we measured participants' AUC in multiple sessions to estimate stable, trait-like tendencies to discount rewards for performing the $N$-back. Specifically, we averaged AUC over three separate discounting sessions for which the intraclass correlation was $0.48,95 \% \mathrm{CI}:(0.22,0.72)$, indicating both stability and variability in intersession discounting.

One possible source of trait-like subjectivity in these prior studies is reward sensitivity. To investigate whether individuals vary in their willingness to perform demanding cognitive tasks because of differences in reward sensitivity, we tested whether AUC predicted the effect of offer amount (reward) on SV representations in the VS and amygdala, two functionally coupled regions which have been shown to reflect trait reward sensitivity (Beck et al., 2009; Hariri, 2009; Plichta and Scheres, 2014). Specifically, we fit hierarchical models to test whether AUC interacted with first-offer amount in predicting average BOLD signal during the valuation period $(6-8 \mathrm{~s}$ after first offer onset). In both the left $\left(B=14.5 \times 10^{-2} ; p=5.3 \times 10^{-4}\right)$ and right $\left(B=15.6 \times 10^{-2} ; p=8.1 \times 10^{-4}\right) \mathrm{VS}$, and in the left $(B=$ $\left.11.0 \times 10^{-2} ; p=0.028\right)$ and right amygdala $\left(B=18.3 \times 10^{-2} ; p=\right.$ $0.0014)$, activity was positively predicted by the interaction of AUC and amount. This AUC-amount interaction implies that high AUC participants were more willing to perform the high-load $N$-back because they were more sensitive to increasing reward amounts. A plot of time courses pursuant to first offer presentation reveals that this interaction is driven by more positive deflections for $\$ 3$ and $\$ 4$ offers, in both sets of regions, for high versus low AUC participants (Fig. 6A). We also found evidence that higher AUC is related to steeper effects of amount on SV in a complementary analysis. Namely, we fit models with amount and load jointly predicting activity in those ROIs reliably tracking SV for each participant, and then tested whether AUC predicted individual differences in fitted amount effects. In both the left $\left(B=2.41 \times 10^{-2}, p=\right.$ $0.0088)$ and right VS $\left(B=2.57 \times 10^{-2}, p=0.059\right.$, trending $)$, and left $\left(B=1.85 \times 10^{-2}, p=0.088\right.$, trending $)$ and right amygdala $\left(B=3.01 \times 10^{-2}, p=0.0072\right)$, amount effects were positively predicted by mean, cross-session AUC (Fig. 6). Note that excluding the high amount effect participant $\left(B_{\mathrm{Vs}}\right.$ Amount Effect $=0.24$; Fig. 6B) did not attenuate the relationship between AUC and amount effects for the remaining participants in either the left $\left(B=2.41 \times 10^{-2}, p=0.0088\right)$ or right VS $\left(B=1.35 \times 10^{-2}, p=\right.$ $0.043)$. We should note, however, that these and subsequent individual difference results should be interpreted with caution given our relatively small sample.

\section{Cognitive demand encoding in AI and dACC reflects working memory performance}

Beyond trait reward sensitivity, we further considered the possibility that subjective effort costs may be related to cognitive task performance. Relationships between SV representation and N-back performance seemed especially likely given that performance correlated with discounting in the present data. Also, one recent study found that subjective effort closely tracked task performance errors (Dunn et al., 2017). Thus, we tested whether average $N$-back performance (the sensitivity index $d^{\prime}$ ) predicted the effect of load on SV representations. We focused on the AI and AACC as these regions have been implicated previously by multiple lines of evidence, including involvement in attention and control modulation (Dosenbach et al., 2006), error awareness and processing (Klein et al., 2007), decisionmaking and learning about physical effort costs (Prévost et al., 2010; Skvortsova et al., 2017), and, in the case of the AI, self-reported cognitive effort ratings (Otto et al., 2014). As above, we also tested the full interaction of load-specific performance and load in predicting 
A

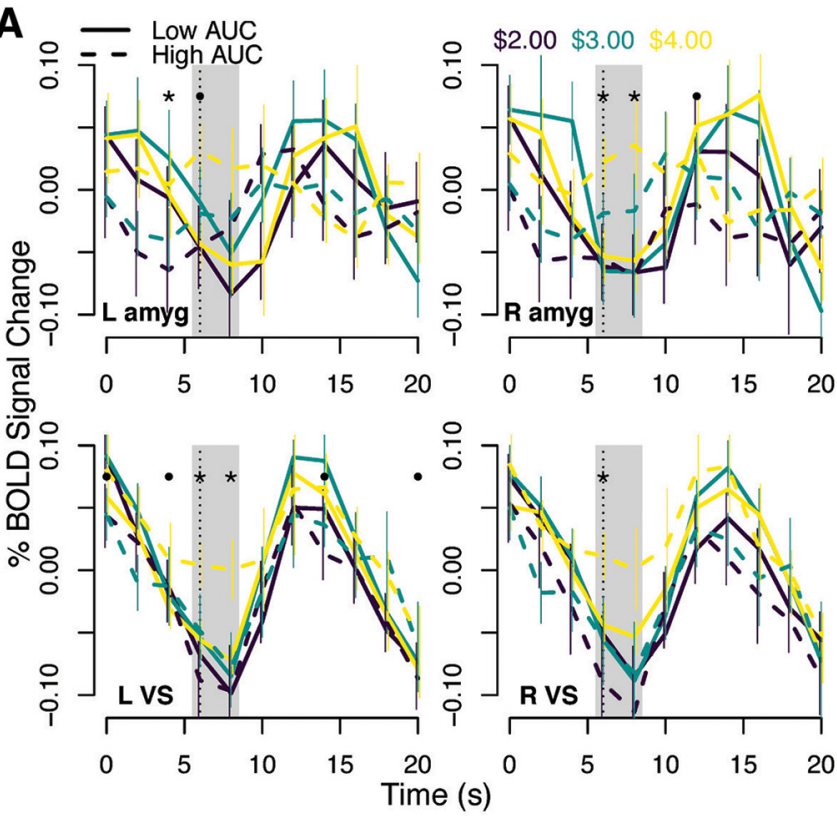

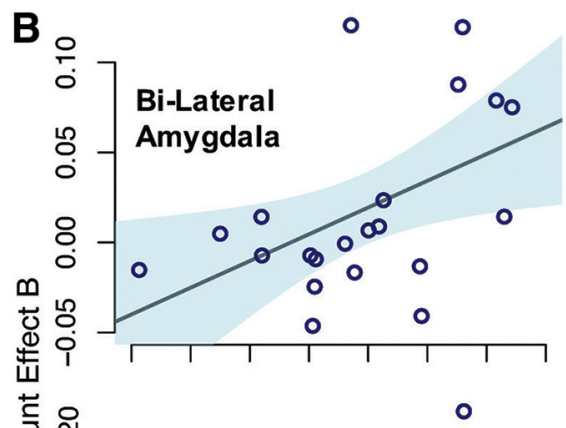

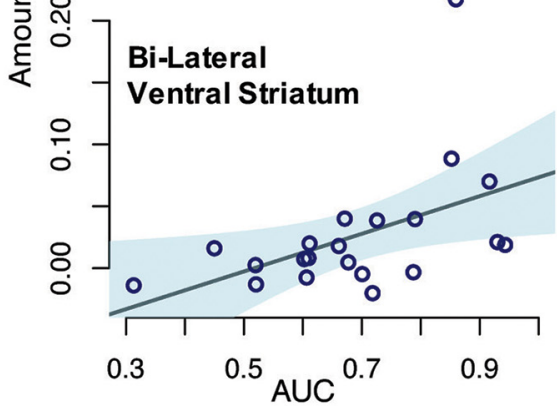

Figure 6. Trait willingness to select high cognitive load for reward varies with reward sensitivity. $\boldsymbol{A}$, Residual time courses in a priori ROls, averaged by first offer amount and divided by above (High) or below median (Low) AUC. Error bars reflect SEM across participants. Reliability of the interaction between AUC and first offer amount in separate hierarchical multiple regression (trials nested within participants) at each time point indicated by ${ }^{*} p<0.05$ and $\cdot p<0.075$. Gray region highlights $6-8 \mathrm{~s}$ after first offer onset; vertical dashed line indicates second offer onset. $\boldsymbol{B}$, Cross-session AUC predicts the average amount effect on mean activity at $6-8 \mathrm{~s}$ following first offer onset in both the bilateral ventral striatum $\left(B=2.49 \times 10^{-2}, p=0.027\right)$ and bilateral amygdala $\left(B=2.43 \times 10^{-2}, p=0.023\right)$. Shaded regions show $95 \% \mathrm{Cl}$.

BOLD signal $6-8 \mathrm{~s}$ after first offer onset. In both the left $\mathrm{AI}(B=$ $\left.1.15 \times 10^{-2} ; p=0.023\right)$ and right $\mathrm{AI}\left(B=8.71 \times 10^{-3} ; p=0.056\right)$ and the $\operatorname{dACC}\left(-2,16,46 ; B=2.62 \times 10^{-2} ; p=0.0067\right)$, we found reliable, positive interactions indicating stronger load effects on BOLD signal for increasingly bad $N$-back performance. These interactions support the hypothesis that predicted task failure is integrated into subjective effort cost representations via the $\mathrm{AI}$ and dACC.

To investigate the nature of those interactions, we again used individual participants' task load effect estimates from a model in which valuation period activity was jointly predicted by task load and amount separately, and then tested whether average $d^{\prime}$ (across all $N$-back levels) predicted individual differences in load effects. A trend-level result in the bilateral AI (Fig. $7 ; B=2.11 \times 10^{-2} ; p=0.085$ ) supported the interpretation that participants with the worst average $N$-back performance tended to show the strongest load effects during the valuation period (activity decreasing with increasing load). The same analysis in the dACC was inconclusive $(p=0.48)$, perhaps reflecting reduced power from collapsing across $N$-back levels and choice trials.

\section{Cognitive effort discounting does not merely reflect task performance}

Although participants were promised payment contingent on merely repeating the $N$-back tasks of their choosing (not on performance), it is possible that decision-making was primarily driven by anticipated likelihood of successful performance at a given load level. In other words, an alternative interpretation of the relationship between $N$-back performance and SV representations is that, rather than reflecting effort discounting and SV encoding per se, apparent SV encoding instead reflected concerns about performing well. This alternative account is plausible given that $N$-back performance covaried negatively with task load, and positively with SV. To test this alternative account, we estimated

\section{Bi-Lateral Anterior Insula}

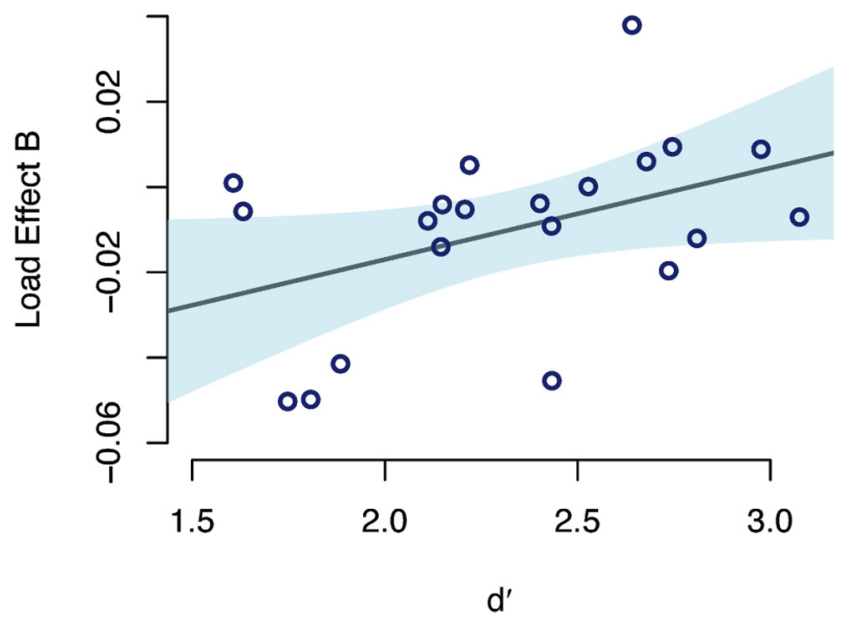

Figure 7. Higher mean $N$-back performance (measured by sensitivity index $d^{\prime}$ ) predicts shallower load effects in bilateral Al across participants $\left(B=2.11 \times 10^{-2} ; p=0.085\right)$. The shaded region shows $95 \% \mathrm{Cl}$.

hierarchical multiple regression models to determine whether valuation period activity in the VmPFC and VS was predicted by SV, controlling for load-level-specific $N$-back performance, and vice versa. The results clearly reject the alternative, performancebased interpretation. In the VS, $N$-back performance did not reliably predict BOLD signal variation (both $p$ values $>0.74$ ), whereas SV did, controlling for performance (left VS: $B=3.76 \times$ $10^{-2}, p=0.018$; right VS: $\left.B=3.96 \times 10^{-2}, p=0.057\right)$. In the vmPFC, although $N$-back performance predicted BOLD signal variation (left $B=1.39 \times 10^{-2}, p=0.055$ and right vmPFC $B=$ $1.42 \times 10^{-2}, p=0.046$, from Levy and Glimcher, 2012; $B=$ $2.70 \times 10^{-2}, p=0.020$, from Bartra et al., 2013), SV was also a 


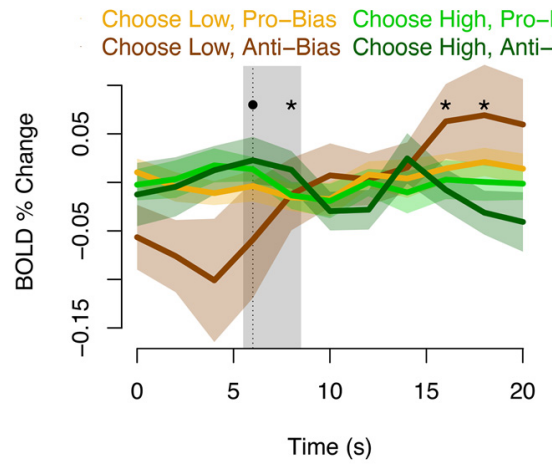

Figure 8. Mean residual time courses at vmPFC loci, averaged by whether participants chose the high or low-demand offer and whether the low-demand offer amount biased them toward the high- or low-demand offer. Error bands reflect SEM across participants. Reliability of choicebias effects in separate, fully random hierarchical multiple regressions at each time point indicated by ${ }^{*} p<0.05$, and $\cdot p<0.075$. Based on a Choose Low, Pro-Bias $<$ Choose Low, Anti-Bias $<$ Choose High, Pro-Bias $<$ Choose High, Anti-Bias coding scheme.

significant predictor, controlling for performance in both the left $\left(B=1.78 \times 10^{-2}, p=0.026\right)$ and right $\operatorname{vmPFC}(B=1.79 \times$ $10^{-2}, p=0.031$, from Levy and Glimcher, 2012, and $B=2.18 \times$ $10^{-2}, p=0.063$ from Bartra et al., 2013). These results support that although anticipated performance partially determines discounting, SV, and neural representations of SV, SV representations further reflect other state and trait factors (e.g., workingmemory load and reward sensitivity) determining willingness to expend cognitive effort.

\section{Trialwise value signals in the vmPFC predict subsequent choice}

In addition to stable, trait subjectivity, valuation may also involve state variation, from factors such as satiety or fatigue that can influence motivation (Hare et al., 2014; Rudorf and Hare, 2014). Although we did not explicitly manipulate state motivation, noncatch trials were close enough to indifference for choice to be sensitive to spontaneous trial-to-trial fluctuations in motivation. Thus, trial-level differences in cognitive or neural state may alter SV representations with consequences for choice behavior. To test for such effects, we examined whether trialwise variation in SV representations predicted choice; specifically, whether valuation period signal predicted choice behavior, above and beyond the bias $(\gamma)$ imposed by the second offer. We focused our analysis on the VS and vmPFC, given clear SV tracking in these regions, and also prior literature implicating vmPFC both in state incentive motivation, and causally determining choice (Hare et al., 2009, 2011, 2014; Rudebeck and Murray, 2014; San-Galli et al., 2018).

During the valuation period, we found that lower vmPFC activity predicted choice of the low-load option, and that this effect was most pronounced on anti-bias trials (when participants do not select the option expected to have the higher SV; or in this case, when the offer was designed to bias choice of the high-load offer but participants still selected the low-load option). A hierarchical model with trials and three vmPFC ROIs nested within participants revealed that this interaction of choice and bias significantly predicted mean vmPFC activity during the valuation period $\left(B=3.57 \times 10^{-2} ; p=0.0072\right)$. Thus, even before participants knew what the low-load (1-back) offer amount would be, trialwise variation in vmPFC activity predicted subsequent choice (Fig. 8). Notably, this pattern was not observed in either the right $\left(B=1.29 \times 10^{-2} ; p=0.57\right)$ or left VS
$\left(B=2.48 \times 10^{-2} ; p=0.22\right)$, despite the VS otherwise covarying with SV. Stronger coupling of choice to vmPFC activity is consistent with the hypothesis that although both VS and vmPFC are part of a distributed valuation network, the vmPFC serves as a final common node incorporating state motivation during decision-making (Levy and Glimcher, 2012). As shown in Figure 8 , the interaction of choice and bias also reliably predicted BOLD signal later in trials with the opposite sign: activity was higher on trial in which participants selected the low-effort option. Although it is possible that this reflects interesting post-decisional processes, the timing of this the interaction, pursuant to presentation of two offers and choice commitment precludes unambiguous interpretation of this effect.

\section{Discussion}

Decisions involving cognitive effort evaluation are reflected in demand avoidance (Kool et al., 2010; Schouppe et al., 2014b) and effort discounting (Botvinick et al., 2009; Dixon and Christoff, 2012; Westbrook et al., 2013; Massar et al., 2015; Chong et al., 2017). Yet it is unclear where and how cognitive effort costs and benefits are encoded in the brain. In this study, we used fMRI as participants decided between a high-load working memory task for more money, or a low-load task for less money, to identify the neural encoding of cognitive effort-discounted SV. Our results provide the first direct evidence that a domain-general valuation network, centered on the vmPFC and VS, tracks SV during decision-making about cognitive effort. This result closely links cognitive effort with other cost domains such as delay, risk, and physical effort (Levy and Glimcher, 2012; Bartra et al., 2013).

Earlier studies have shown valuation regions encoding reward signals modulated by prior or anticipated cognitive demands (Botvinick et al., 2009; Satterthwaite et al., 2012; Schmidt et al., 2012; Schouppe et al., 2014a; Vassena et al., 2014; Dobryakova et al., 2017; Nagase et al., 2018). These results imply that the valuation network should also encode SV during cognitive effortbased decision-making. Yet, to date, this has not been shown. In fact, the only two studies directly examining cognitive effortdiscounted SV (Massar et al., 2015; Chong et al., 2017) identified regions outside the valuation network, raising the possibility that decisions about cognitive effort involve fundamentally different mechanisms than other cost domains. Indeed, these studies found that SV was encoded primarily in frontoparietal regions typically associated with cognitive control itself, including the dlPFC and IPS.

Our results suggest a reinterpretation of these prior studies, namely that frontoparietal regions track shared variance between SV regressors and choice difficulty. Note that we are agnostic as to whether frontoparietal ROIs encode SV when offers are directly compared. The dACC in particular, e.g., encodes SV differences between competing alternatives in both reward and physical effort demands (Klein-Flügge et al., 2016). However, it may be that these regions are recruited primarily for careful comparison between close alternatives. Such regions would not, therefore, reliably track first-offer SV in our design, which was focused on valuation of a single offer in isolation.

It is possible, however, to mistakenly implicate regions primarily tracking difficulty with SV encoding if the two are correlated. Consider frontoparietal cognitive control regions recruited to focus attention during difficult value-based discriminations (Jimura et al., 2018). Such regions could be recruited as a function of value differences, without computing SV per se. Thus, it is critical to also test for SV encoding when controlling for decision difficulty, as we did by: (1) examining BOLD response to single 
offers in isolation, (2) balancing offers so that participants prefer the high-demand/high-benefit option on approximately half the trials, and (3) fully-crossing offer attributes to decorrelate SV and difficulty. With this approach, we doubly dissociated a valuation network reliably tracking first-offer SV, and a frontoparietal network reliably tracking decision difficulty.

Our conclusions align closely with a recent physical effort study, which found that the vmPFC encoded SV while the ACC tracked decision difficulty (Hogan et al., 2018). Interestingly, despite coming to the same conclusions as our own study, this study used methods more like those used in the prior cognitive effort studies. Namely, they also examined value encoding during simultaneous offer presentation, and regressed BOLD signal on the SV difference between competing offers. So how could they come to different conclusions? For one, this study (like Study 1) balanced high- and low-cost offer selection and regressed out reaction time to control for conflict-related difficulty. However, unlike Study 1, only effort costs varied (no rewards were offered). Perhaps, therefore, what prevented the prior cognitive effort studies from implicating the valuation network was the complexity of the decision space and uncertainty about encoding across two attributes and two alternatives at any given time point. By holding reward constant, the physical effort study benefitted from greater certainty about what choice dimensions were encoded during offer comparison, whereas in our study, the presentation of a single alternative also simplified the decision space.

Beyond showing that a domain-general valuation network tracks SV, we further show that this network scales both positively with amount, and negatively with cognitive load. This result was critical to demonstrate that the network encoded SV rather than merely correlating with a single dimension, such as rewards on offer; a result that is already well established within the vmPFC and VS (Bartra et al., 2013). On an individual ROI basis, the AI and AACC demonstrated sensitivity to cognitive load but not reward amount. This could reflect limited power to detect reward encoding: the dACC has elsewhere been shown to track both physical effort costs and reward amount (Harris and Lim, 2016; Klein-Flügge et al., 2016). Interestingly, however, numerous lines of evidence also suggest a somewhat more specialized role for these regions in processing cognitive effort costs including involvement in control modulation (Dosenbach et al., 2006), error awareness and processing (Klein et al., 2007), decisionmaking about physical effort (Croxson et al., 2009; Prévost et al., 2010; Kennerley et al., 2011; Kurniawan et al., 2013; Skvortsova et al., 2014), self-reported cognitive effort (in the AI; Otto et al., 2014), and learning about cognitive effort costs (Botvinick, 2007; Nagase et al., 2018).

Our results are thus consistent with the overarching hypothesis that the dACC and AI specialize in processing effort cost information in the service of SV computation. This interpretation is bolstered by additional findings that individual differences in $\mathrm{N}$-back performance modulate cost encoding (load effects) within $\mathrm{dACC}$ and $\mathrm{AI}$, suggesting that these regions may track the subjective difficulty (expected performance decline) associated with higher cognitive load. With regard to the dACC in particular, an influential account, known as expected value of control (EVC), implicates this region in regulating control as a function of expected reward benefits and effort costs (Shenhav et al., 2013). Our results are consistent with the EVC, in demonstrating that information about both costs and benefits are represented in the ACC proper during offer valuation. Moreover, the EVC account also predicts increased dACC involvement when decisions are more difficult. Our data confirm this prediction by showing higher dACC activity when participants decide between offers that were close in value versus when offers were far apart. In fact, the dACC ROI was one of the few to reliably track to both prospective cognitive load and decision difficulty, a pattern of results that is uniquely predicted by the EVC hypothesis.

Our results also elucidate factors driving subjectivity in SV. First, larger amount effects in the VS and amygdala predicted shallower discounting across participants. This result is consistent with the close structural and functional connectivity of these regions for reward processing (Cardinal et al., 2002) and evidence that they reflect trait reward sensitivity (Hariri et al., 2006; Beck et al., 2009; Plichta and Scheres, 2014). Second, trialwise vmPFC representations of SV predict choice. Specifically, low-load choices were anticipated by below-average vmPFC signal during the valuation period, and this was particularly true on when offers biased high-load offer selection. This finding is consistent with the hypothesis that state variation in SV, as encoded by the vmPFC, determines momentary preference (Hare et al., 2009, 2011, 2014; Rudebeck and Murray, 2014), current motivational state (Bouret and Richmond, 2010), and willingness to perform effortful instrumental tasks (San-Galli et al., 2018).

Our a priori ROI approach provided increased statistical power, while protecting against false-positives that are of greater concern in whole-brain voxelwise analyses. Nevertheless, one limitation was that we did not investigate representational heterogeneity across the larger cortical regions identified in the prior cognitive effort studies. Complementary whole-brain analyses did not identify any clusters overlapping regions identified in the prior studies. Yet, this null result may reflect false negatives, or missed dynamics in other parts of regions identified in Study 1 and Study 2. Future work is also needed to elucidate the nature of value signals; e.g., to discern whether valuation regions are encoding value as opposed to subjective relief when prospecting about low-effort options. Finally, our study is limited by a relatively small sample size and low-power. This concern is particularly relevant for individual difference analyses including subjectivity effects in amount and load encoding. Although our results conform to strong prior predictions, replication studies with higher-power are needed. Nevertheless, these results provide clear evidence that a domain-general valuation network encodes cognitive effort-discounted SV, while also suggesting how other components (e.g., amygdala, dACC, AI) might be particularly sensitive to individual difference variables.

Recent theoretical and empirical work highlights that subjective cognitive effort costs modulate cognitive control demand avoidance, and contribute to psychopathology (Gold et al., 2015; Culbreth et al., 2016). By implicating a domain-general valuation network in cognitive effort-related SV encoding, our results yield considerable leverage in understanding the mechanisms by which decisions about cognitive effort are made. Moreover, they point to coherent neural targets for interventions to enhance cognitive motivation in impaired populations.

\section{References}

Aridan N, Malecek NJ, Poldrack RA, Schonberg T (2019) Neural correlates of effort-based valuation with prospective choices. Neuroimage 185:446454.

Bartra O, McGuire JT, Kable JW (2013) The valuation system: a coordinatebased meta-analysis of BOLD fMRI experiments examining neural correlates of subjective value. Neuroimage 76:412-427.

Basten U, Biele G, Heekeren HR, Fiebach CJ (2010) How the brain integrates costs and benefits during decision making. Proc Natl Acad Sci U S A 107:21767-21772.

Beck A, Schlagenhauf F, Wüstenberg T, Hein J, Kienast T, Kahnt T, Schmack 
K, Hägele C, Knutson B, Heinz A, Wrase J (2009) Ventral striatal activation during reward anticipation correlates with impulsivity in alcoholics. Biol Psychiatry 66:734-742.

Benjamini Y, Hochberg Y (1995) Controlling the false discovery rate: a practical and powerful approach to multiple testing. J Royal Stat Soc B 57:289-300.

Botvinick MM (2007) Conflict monitoring and decision making: reconciling two perspectives on anterior cingulate function. Cogn Affect Behav Neurosci 7:356-366.

Botvinick MM, Braver TS, Barch DM, Carter CS, Cohen JD (2001) Conflict monitoring and cognitive control. Psychol Rev 108:624-652.

Botvinick MM, Huffstetler S, McGuire JT (2009) Effort discounting in human nucleus accumbens. Cogn Affect Behav Neurosci 9:16-27.

Boureau YL, Sokol-Hessner P, Daw ND (2015) Deciding how to decide: self-control and meta-decision making. Trends Cogn Sci 19:700-710.

Bouret S, Richmond BJ (2010) Ventromedial and orbital prefrontal neurons differentially encode internally and externally driven motivational values in monkeys. J Neurosci 30:8591-8601.

Brainard DH (1997) The psychophysics toolbox. Spat Vis 10:433-436.

Braver TS, Cohen JD, Nystrom LE, Jonides J, Smith EE, Noll DC (1997) A parametric study of prefrontal cortex involvement in human working memory. Neuroimage 5:49-62.

Cardinal RN, Parkinson JA, Hall J, Everitt BJ (2002) Emotion and motivation: the role of the amygdala, ventral striatum, and prefrontal cortex. Neurosci Biobehav Rev 26:321-352.

Chapman GB, Johnson EJ (1999) Anchoring, activation, and the construction of values. Organ Behav Hum Decis Process 79:115-153.

Chong TT, Apps MA, Giehl K, Sillence A, Grima LL, Husain M (2017) Neurocomputational mechanisms underlying subjective valuation of effort costs. PLoS Biol 15:e1002598.

Cohen R, Lohr I, Paul R, Boland R (2001) Impairment of attention and effort among patients with major affective disorders. J Neuropsychiatry Clin Neurosci 13:385-395.

Cox RW (1996) AFNI: software for analysis and visualization of functional magnetic resonance neuroimages. Comput Biomed Res 29:162-173.

Croxson PL, Walton ME, O’Reilly JX, Behrens TE, Rushworth MF (2009) Effort-based cost-benefit valuation and the human brain. J Neurosci 29: 4531-4541.

Culbreth A, Westbrook A, Barch D (2016) Negative symptoms are associated with an increased subjective cost of cognitive effort. J Abnorm Psychol 125:528-536.

Dixon ML, Christoff K (2012) The decision to engage cognitive control is driven by expected reward-value: neural and behavioral evidence. PLoS One 7:e51637.

Dobryakova E, Jessup RK, Tricomi E (2017) Modulation of ventral striatal activity by cognitive effort. Neuroimage 147:330-338.

Dosenbach NU, Visscher KM, Palmer ED, Miezin FM, Wenger KK, Kang HC, Burgund ED, Grimes AL, Schlaggar BL, Petersen SE (2006) A core system for the implementation of task sets. Neuron 50:799-812.

Dunn TL, Inzlicht M, Risko EF (2017) Anticipating cognitive effort: roles of perceived error-likelihood and time demands. Psychol Res. Advance online publication. Retrieved November 13, 2017. doi: 10.1007/s00426-0170943-x.

Eickhoff SB, Stephan KE, Mohlberg H, Grefkes C, Fink GR, Amunts K, Zilles K (2005) A new SPM toolbox for combining probabilistic cytoarchitectonic maps and functional imaging data. Neuroimage 25:1325-1335.

Fonov V, Evans AC, Botteron K, Almli CR, McKinstry RC, Collins DL (2011) Unbiased average age-appropriate atlases for pediatric studies. Neuroimage 54:313-327.

Gelman A, Hill J (2007) Data analysis using regression and multilevel/hierarchical models. New York: Cambridge.

Gold JM, Waltz JA, Frank MJ (2015) Effort cost computation in schizophrenia: a commentary on the recent literature. Biol Psychiatry 78:747-753.

Hare TA, Camerer CF, Rangel A (2009) Self-control in decision-making involves modulation of the vmPFC valuation system. Science 324:646648.

Hare TA, Malmaud J, Rangel A (2011) Focusing attention on the health aspects of foods changes value signals in vmPFC and improves dietary choice. J Neurosci 31:11077-11087.

Hare TA, Hakimi S, Rangel A (2014) Activity in dlPFC and its effective connectivity to vmPFC are associated with temporal discounting. Front Neurosci 8:50.
Hariri AR (2009) The neurobiology of individual differences in complex behavioral traits. Annu Rev Neurosci 32:225-247.

Hariri AR, Brown SM, Williamson DE, Flory JD, de Wit H, Manuck SB (2006) Preference for immediate over delayed rewards is associated with magnitude of ventral striatal activity. J Neurosci 26:13213-13217.

Harris A, Lim SL (2016) Temporal dynamics of sensorimotor networks in effort-based cost-benefit valuation: early emergence and late net value integration. J Neurosci 36:7167-7183.

Hartmann MN, Hager OM, Tobler PN, Kaiser S (2013) Parabolic discounting of monetary rewards by physical effort. Behav Processes 100:192-196.

Hogan PS, Galaro JK, Chib VS (2018) Roles of ventromedial prefrontal cortex and anterior cingulate in subjective valuation of prospective effort. Cereb Cortex. Advance online publication. Retrieved December 12, 2018. doi:10.1093/cercor/bhy310.

Hopstaken JF, van der Linden D, Bakker AB, Kompier MA (2015) The window of my eyes: task disengagement and mental fatigue covary with pupil dynamics. Biol Psychol 110:100-106.

Hosking JG, Cocker PJ, Winstanley CA (2014) Dissociable contributions of anterior cingulate and basolateral amygdala on a rodent cost/benefit decision-making task of cognitive effort. Neuropsychopharmacology 39:1558-1567.

Hunt LT, Kolling N, Soltani A, Woolrich MW, Rushworth MF, Behrens TEJ (2012) Mechanisms underlying cortical activity during value-guided choice. Nat Neurosci 15:470-476. S1-S3.

Jimura K, Chushak MS, Westbrook A, Braver TS (2018) Intertemporal decision-making involves prefrontal control mechanisms associated with working memory. Cereb Cortex 28:1105-1116.

Kennerley SW, Behrens TE, Wallis JD (2011) Double dissociation of value computations in orbitofrontal and anterior cingulate neurons. Nat Neurosci 14:1581-1589.

Kirchner WK (1958) Age differences in short-term retention of rapidly changing information. J Exp Psychol 55:352-358.

Klein TA, Endrass T, Kathmann N, Neumann J, von Cramon DY, Ullsperger M (2007) Neural correlates of error awareness. Neuroimage 34:17741781.

Klein-Flügge MC, Kennerley SW, Friston K, Bestmann S (2016) Neural signatures of value comparison in human cingulate cortex during decisions requiring an effort-reward trade-off. J Neurosci 36:10002-10015.

Kool W, McGuire JT, Rosen ZB, Botvinick MM (2010) Decision making and the avoidance of cognitive demand. J Exp Psychol Gen 139:665-682.

Kouneiher F, Charron S, Koechlin E (2009) Motivation and cognitive control in the human prefrontal cortex. Nat Neurosci 12:939-945.

Kurniawan IT, Guitart-Masip M, Dayan P, Dolan RJ (2013) Effort and valuation in the brain: the effects of anticipation and execution. J Neurosci 33:6160-6169.

Kurzban R, Duckworth A, Kable JW, Myers J (2013) An opportunity cost model of subjective effort and task performance. Behav Brain Sci 36:661679.

Levy DJ, Glimcher PW (2012) The root of all value: a neural common currency for choice. Curr Opin Neurobiol 22:1027-1038.

Manohar SG, Chong TT, Apps MAJ, Batla A, Stamelou M, Jarman PR, Bhatia KP, Husain M (2015) Reward pays the cost of noise reduction in motor and cognitive control. Curr Biol 25:1707-1716.

Massar SA, Libedinsky C, Weiyan C, Huettel SA, Chee MW (2015) Separate and overlapping brain areas encode subjective value during delay and effort discounting. Neuroimage 120:104-113.

Miezin FM, Maccotta L, Ollinger JM, Petersen SE, Buckner RL (2000) Characterizing the hemodynamic response: effects of presentation rate, sampling procedure, and the possibility of ordering brain activity based on relative timing. Neuroimage 11:735-759.

Miller EK (2000) The prefrontal cortex and cognitive control. Nat Rev Neurosci 1:59-65.

Musslick S, Jang SJ, Shvartsman M, Shenhav A, Cohen JD (2018) Constraints associated with cognitive control and the stability-flexibility dilemma. Proceedings of the 40th Annual Meeting of the Cognitive Science Society. Madison, WI.

Myerson J, Green L, Warusawitharana M (2001) Area under the curve as a measure of discounting. J Exp Anal Behav 76:235-243.

Nagase AM, Onoda K, Clifford Foo J, Haji T, Akaishi R, Yamaguchi S, Sakai K, Morita K (2018) Neural mechanisms for adaptive learned avoidance of mental effort. J Neurosci 38:2631-2651.

Otto T, Zijlstra FR, Goebel R (2014) Neural correlates of mental effort eval- 
uation: involvement of structures related to self-awareness. Soc Cogn Affect Neurosci 9:307-315.

Padoa-Schioppa C (2011) Neurobiology of economic choice: a good-based model. Annu Rev Neurosci 34:333-359.

Plichta MM, Scheres A (2014) Neuroscience and biobehavioral reviews. Neurosci Biobehav Rev 38:125-134.

Pochon JB, Riis J, Sanfey AG, Nystrom LE, Cohen JD (2008) Functional imaging of decision conflict. J Neurosci 28:3468-3473.

Prévost C, Pessiglione M, Météreau E, Cléry-Melin ML, Dreher JC (2010) Separate valuation subsystems for delay and effort decision costs. J Neurosci 30:14080-14090.

Rangel A, Camerer C, Montague PR (2008) A framework for studying the neurobiology of value-based decision making. Nat Rev Neurosci 9:545-556.

Rudebeck PH, Murray EA (2014) The orbitofrontal oracle: cortical mechanisms for the prediction and evaluation of specific behavioral outcomes. Neuron 84:1143-1156.

Rudorf S, Hare TA (2014) Interactions between dorsolateral and ventromedial prefrontal cortex underlie context-dependent stimulus valuation in goal-directed choice. J Neurosci 34:15988-15996.

San-Galli A, Varazzani C, Abitbol R, Pessiglione M, Bouret S (2018) Primate ventromedial prefrontal cortex neurons continuously encode the willingness to engage in reward-directed behavior. Cereb Cortex 28:73-89.

Satterthwaite TD, Ruparel K, Loughead J, Elliott MA, Gerraty RT, Calkins ME, Hakonarson H, Gur RC, Gur RE, Wolf DH (2012) Being right is its own reward: load and performance related ventral striatum activation to correct responses during a working memory task in youth. Neuroimage 61:723-729.

Schmidt L, Lebreton M, Cléry-Melin ML, Daunizeau J, Pessiglione M (2012) Neural mechanisms underlying motivation of mental versus physical effort. PLoS Biol 10:e1001266.

Schouppe N, Demanet J, Boehler CN, Ridderinkhof KR, Notebaert W (2014a) The role of the striatum in effort-based decision-making in the absence of reward. J Neurosci 34:2148-2154.

Schouppe N, Ridderinkhof KR, Verguts T, Notebaert W (2014b) Contextspecific control and context selection in conflict tasks. Acta Psychologica 146:63-66.
Shenhav A, Botvinick MM, Cohen JD (2013) The expected value of control: an integrative theory of anterior cingulate cortex function. Neuron 79:217-240.

Shenhav A, Straccia MA, Cohen JD, Botvinick MM (2014) Anterior cingulate engagement in a foraging context reflects choice difficulty, not foraging value. Nat Neurosci 17:1249-1254.

Shenhav A, Musslick S, Lieder F, Kool W, Griffiths TL, Cohen JD, Botvinick MM (2017) Toward a rational and mechanistic account of mental effort. Annu Rev Neurosci 40:99-124.

Siegel JS, Power JD, Dubis JW, Vogel AC, Church JA, Schlaggar BL, Petersen SE (2014) Statistical improvements in functional magnetic resonance imaging analyses produced by censoring high-motion data points. Hum Brain Mapp 35:1981-1996.

Sinha N, Manohar S, Husain M (2013) Impulsivity and apathy in Parkinson's disease. J Neuropsychol 7:255-283.

Skvortsova V, Palminteri S, Pessiglione M (2014) Learning to minimize efforts versus maximizing rewards: computational principles and neural correlates. J Neurosci 34:15621-15630.

Skvortsova V, Degos B, Welter ML, Vidailhet M, Pessiglione M (2017) A selective role for dopamine in learning to maximize reward but not to minimize effort: evidence from patients with Parkinson's disease. J Neurosci 37:6087-6097.

Vassena E, Silvetti M, Boehler CN, Achten E, Fias W, Verguts T (2014) Overlapping neural systems represent cognitive effort and reward anticipation. PLoS One 9:e91008.

Volkow ND, Wang GJ, Newcorn JH, Kollins SH, Wigal TL, Telang F, Fowler JS, Goldstein RZ, Klein N, Logan J, Wong C, Swanson JM (2011) Motivation deficit in $\mathrm{ADHD}$ is associated with dysfunction of the dopamine reward pathway. Mol Psychiatry 16:1147-1154.

Westbrook A, Kester D, Braver TS (2013) What is the subjective cost of cognitive effort? Load, trait, and aging effects revealed by economic preference. PLoS One 8:e68210.

Yarkoni T, Gray JR, Chrastil ER, Barch DM, Green L, Braver TS (2005) Sustained neural activity associated with cognitive control during temporally extended decision making. Brain Res Cogn Brain Res 23:71-84. 